\title{
THE
}

UNIVERSITY

University of Rhode Island

OF RHODE ISLAND

DigitalCommons@URI

\section{Chronic Opioid Use in Women Following Hysterectomy: Patterns and Predictors}

Xuerong Wen

University of Rhode Island, xuerongwen@uri.edu

Jon Kogut

University of Rhode Island, skogut@uri.edu

Hilary Aroke

University of Rhode Island

Lynn E. Taylor

University of Rhode Island, letaylor@uri.edu

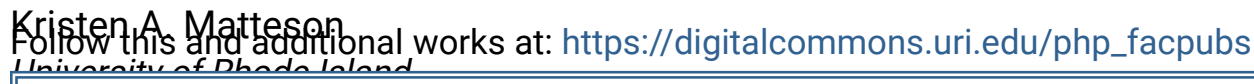

The University of Rhode Island Faculty have made this article openly available.

Please let us know how Open Access to this research benefits you.

This is a pre-publication author manuscript of the final, published article.

Terms of Use

This article is made available under the terms and conditions applicable towards Open Access

Policy Articles, as set forth in our Terms of Use.

\section{Citation/Publisher Attribution}

Wen, $\mathrm{X}$, Kogut, S, Aroke, H, Taylor, L, Matteson, KA. Chronic opioid use in women following hysterectomy: Patterns and predictors. Pharmacoepidemiol Drug Saf. 2020; 29: 493- 503. https://doi.org/10.1002/ pds.4972

Available at: https://doi.org/10.1002/pds.4972 


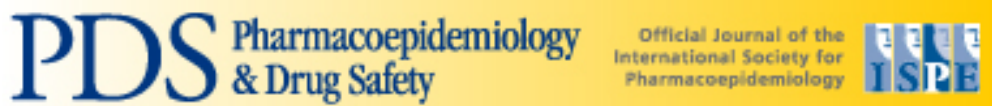

\section{Chronic Opioid Use in Women Following Hysterectomy: Patterns and Predictors}

\begin{tabular}{|c|c|}
\hline Journal: & Pharmacoepidemiology and Drug Safety \\
\hline Manuscript ID & PDS-18-0298.R2 \\
\hline Wiley - Manuscript type: & Original Report \\
\hline $\begin{array}{r}\text { Date Submitted by the } \\
\text { Author: }\end{array}$ & $n / a$ \\
\hline Complete List of Authors: & $\begin{array}{l}\text { Wen, Xuerong; University of Rhode Island - College of Pharmacy, Health } \\
\text { Outcomes, Department of Pharmacy Practice } \\
\text { Kogut, Stephen ; University of Rhode Island - College of Pharmacy, } \\
\text { Health Outcomes, Department of Pharmacy Practice } \\
\text { Aroke, Hilary; University of Rhode Island - College of Pharmacy, Health } \\
\text { Outcomes, Department of Pharmacy Practice } \\
\text { Tayler, Lynn; University of Rhode Island - College of Pharmacy, Health } \\
\text { Outcomes, Department of Pharmacy Practice } \\
\text { Matteson, Kristen; The Warren Alpert Medical School, Brown University., } \\
\text { Obstetrics and Gynecology }\end{array}$ \\
\hline Keywords: & hysterectomy, predictors, chronic opioid use \\
\hline Abstract: & $\begin{array}{l}\text { Background: Most women are prescribed an opioid after hysterectomy. } \\
\text { The objective of this study was to determine the association between } \\
\text { initial opioid prescribing characteristics and chronic opioid use after } \\
\text { hysterectomy. } \\
\text { Methods: This study included women enrolled in a commercial health } \\
\text { plan who had a hysterectomy between July } 01,2010 \text { and March } 31, \\
2015 \text {. We used trajectory models to define chronic opioid use as patients } \\
\text { with the highest probability of having an opioid prescription filled during } \\
\text { the } 6 \text { months post-surgery. We used multivariable logistic regression to } \\
\text { examine the association between initial opioid dispensing (amount } \\
\text { prescribed and duration of treatment) and chronic opioid use after } \\
\text { adjusting for potential confounders. } \\
\text { Results: A total of } 693 \text { of } 50,127 \text { (1.38\%) opioid naïve women met the } \\
\text { criteria for chronic opioid use following hysterectomy. The baseline } \\
\text { variables and initial opioid prescription characteristics predicted the } \\
\text { pattern of long-term opioid use with moderate discrimination (c statistic } \\
=0.70) \text {. Significant predictors of chronic opioid use included initial opioic } \\
\text { daily dose ( } \geq 60 \text { MME vs }<40 \text { MME, aOR: } 1.43,95 \% \text { CI: } 1.14-1.79 \text { ), and } \\
\text { days' supply } 4-7 \text { days vs } 1-3 \text { days, aOR: } 1.28,95 \% \text { CI: } 1.06-1.54 ; \\
\text { days vs } 1-3 \text { days, aOR: } 1.41,95 \% C I: 1.05-1.89 \text { ). Other significant } \\
\text { baseline predictors included older age, abdominal or laparoscopic/robotic } \\
\text { hysterectomy, tobacco use, psychiatric medication use, back pain, and } \\
\text { headache. } \\
\text { Conclusion: Initial opioid prescribing characteristics are associated with } \\
\text { risk of chronic opioid use after hysterectomy. Prescribing lower daily } \\
\text { doses and shorter days' supply of opioids to women after hysterectomy }\end{array}$ \\
\hline
\end{tabular}


may result in lower risk of chronic opioid use.

\section{SCHOLARONE ${ }^{\text {M }}$ \\ Manuscripts}




\section{Chronic Opioid Use in Women following Hysterectomy: Patterns and Predictors}

Xuerong Wen, ${ }^{1}$ PhD, MPH, Stephen Kogut, ${ }^{1}$ PhD, Hilary Aroke,${ }^{1}$ MD, PhD, MPH, Lynn Taylor ${ }^{1}$, MD, Kristen A. Matteson, ${ }^{2} \mathrm{MD}, \mathrm{MPH}$,

1. Health Outcomes Research, Department of Pharmacy Practice, College of Pharmacy, University of Rhode Island.

2. Obstetrics and Gynecology, Women \& Infants Hospital and the Warren Alpert Medical School, Brown University. 


\begin{abstract}
Background: Most women are prescribed an opioid after hysterectomy. The objective of this study was to determine the association between initial opioid prescribing characteristics and chronic opioid use after hysterectomy.

Methods: This study included women enrolled in a commercial health plan who had a hysterectomy between July 01, 2010 and March 31, 2015. We used trajectory models to define chronic opioid use as patients with the highest probability of having an opioid prescription filled during the 6 months post-surgery. We used multivariable logistic regression to examine the association between initial opioid dispensing (amount prescribed and duration of treatment) and chronic opioid use after adjusting for potential confounders.

Results: A total of 693 of 50,127 (1.38\%) opioid naïve women met the criteria for chronic opioid use following hysterectomy. The baseline variables and initial opioid prescription characteristics predicted the pattern of long-term opioid use with moderate discrimination (c statistic $=0.70$ ). Significant predictors of chronic opioid use included initial opioid daily dose ( $\geq 60$ MME vs $<40$ MME, aOR: $1.43,95 \% \mathrm{Cl}: 1.14-1.79)$, and days' supply 4-7 days vs 1-3 days, aOR: 1.28 , $95 \% \mathrm{Cl}: 1.06-1.54 ; \geq 8$ days vs $1-3$ days, aOR: $1.41,95 \% \mathrm{Cl}: 1.05-1.89)$. Other significant baseline predictors included older age, abdominal or laparoscopic/robotic hysterectomy, tobacco use, psychiatric medication use, back pain, and headache.

Conclusion: Initial opioid prescribing characteristics are associated with risk of chronic opioid use after hysterectomy. Prescribing lower daily doses and shorter days' supply of opioids to women after hysterectomy may result in lower risk of chronic opioid use.
\end{abstract}




\section{Introduction}

The rapid increase in the incidence of opioid-related overdoses and deaths has become a major public health crisis in the United States. Overdose and death from prescription opioid overdose increased $400 \%$ in women between 1999 and $2013 .{ }^{1-3}$ For many women, their first exposure to prescription opioids often occurs during the post-operative period, which makes this a potential target for strategies to reduce the risk of chronic opioid use. ${ }^{4-6}$ Several observational studies suggest that surgery is a risk factor for chronic opioid use. ${ }^{5-18}$ Two studies have examined the relationship between the initial opioid prescribing characteristics and chronic opioid use in the postoperative setting and arrived at contradictory conclusions. ${ }^{6,12}$ One study reported that initial exposure to prescription opioids after minor surgery increases the risk of chronic opioid use by $44 \%,{ }^{12}$ while the other suggested that the initial opioid prescribing characteristics, including type of opioid dispensed, days' supply, or daily dose (in morphine milligram equivalents), were not associated with chronic opioid use after cesarean delivery. ${ }^{6}$

Hysterectomy, the most commonly performed non-obstetric surgery among women in the United States, ${ }^{19-21}$ poses a potential risk for chronic opioid use because an estimated $82 \%$ of patients receive an opioid prescription after the hysterectomy surgery. ${ }^{20,22}$ However, the relationship between the initial opioid prescribing characteristics and chronic opioid use after hysterectomy remains largely unknown. The objective of this study was to identify baseline risk factors for chronic opioid use after hysterectomy and examine the association between initial opioid prescribing characteristics after hysterectomy and subsequent chronic use of opioids among opioid naïve women.

\section{Methods}

Data Sources: Study data were derived from the national Optumlnsight Clinformatics Data Mart $^{\mathrm{TM}}$ (Optumlnsight, Eden Prairie, MN). The Optum Clinformatics Data Mart is an administrative health claims database from a large national insurer which includes 
approximately 35 million beneficiaries. The dataset contains transactional reimbursement data for health care utilization including outpatient pharmacy dispensing, and inpatient and outpatient medical claims. ${ }^{23}$ The administrative enrollment file has eligibility information, the outpatient pharmacy file has the national drug code (NDC) for each drug dispensed, and the medical files have the Current Procedural Terminology (CPT) code for medical procedure, and International Classification of Diseases, Ninth Revision, Clinical Modification (ICD-9-CM) code for medical procedure and diagnosis. Patients included in the database had both medical and prescription coverage. The data was used under license agreement between the University of Rhode Island and Optumlnsight Inc.

Study Population: This study included women ( $\geq 18$ years) who had a hysterectomy between July 01, 2010 and March 30, 2015. Women were required to have at least 6 months of continuous enrollment prior to hysterectomy. To identify an opioid-naïve study cohort, we excluded women with a diagnosis of opioid use disorders or any opioids filled 6 months before the date of the hysterectomy (index date) (eTable 1). The hysterectomy procedures were identified using ICD-9-CM procedure and CPT codes from both the inpatient and outpatient claims (eTable 1). ${ }^{21,24}$ We excluded patients who had a radical hysterectomy and patients for whom we could not rule-out a cancer diagnosis which was defined as $\geq 2$ separate medical claims with a cancer diagnosis occurred $\geq 42$ days apart or receipt of chemotherapy, radiation, or other cancer-related surgery (eTable 1). ${ }^{25}$ Women who filled at least one opioid prescription at a retail pharmacy within 7 days of discharge were considered exposed to prescription opioids and included in the final study cohort. The 7-day window was based on the assumption that an opioid prescription filled by an opioid naïve individual during this period was likely used to treat acute pain after hysterectomy. This study was approved by the University of Rhode Island Institutional Review Board (IRB\#957873-2).

Exposure to opioids: Opioid prescriptions were identified using national drug codes (NDC) from the outpatient pharmacy claims. Opioid prescriptions were classified as hydrocodone, 
oxycodone and others (including codeine, fentanyl, hydromorphone, meperidine, morphine, pentazocine, tapentadol, and tramadol). Duration of the initial prescription was categorized as 1$3,4-7, \geq 8$ days. We calculated the morphine milligram equivalent (MME) for the initial opioid prescription using the Center for Disease Control (CDC) conversion Tables (2016 version). The average daily MME dose was categorized into tertiles ( $\leq 40 \mathrm{mg} / \mathrm{day}, 40.50-58.9 \mathrm{mg} / \mathrm{day}$, and $\geq 60 \mathrm{mg} /$ day).

Outcome Assessment: We used trajectory models to generate our dichotomous primary study outcome as chronic opioid use after hysterectomy present or absent. ${ }^{11}$ The trajectory models allow the use of observed longitudinal data to determine distinct opioid prescription filling patterns in the study population during the six months period after hysterectomy. This approach classifies patients into groups with similar opioid prescription filling patterns during follow-up without relying on a prespecified cutoff value for the definition of chronic opioid use. ${ }^{26,27}$ In order to classify the trajectory groups for opioid use during the defined follow-up window (6 months after hysterectomy), we first generated 6 dichotomous variables to indicate if a study participant filled a prescription of an opioid analgesic during each of 6 consecutive 30-day follow-up periods, e.g., month 1 is from 7 to 37 days, month 2 is from 37 to 67 days, and month 6 is from 157 to 187 days. ${ }^{11}$ We then modeled these 6 binary indicators of opioid use in each 30 -day follow-up period as a longitudinal response in a logistic group-based trajectory. ${ }^{28,29}$ Using the trajectory model, we estimated the probability of membership of patients in each group, and the probability of filling an opioid prescription over time as a smooth function of time. The model was fitted using 2 to 5 opioid exposure groups and the number of groups was chosen based on the value of the Bayesian Information Criterion. ${ }^{30}$ In each group, a third-order polynomial (including linear, squared, and cubic terms) of time were used to model the probability of filling a prescription for opioids. Patients were assigned to the trajectory group in which they had the highest probability of membership. Based on the model results, the trajectory group with the highest probability of filling any opioid prescription beyond the initial prescription was defined as 
"chronic opioid users". All other trajectory groupings were classified as non-chronic opioid users. The percentage of patients who filled an opioid prescription was reported in each trajectory for every consecutive 30-day follow-up period.

Potential Predictors of Chronic Opioid Use: We collected data on potential confounding variables that may be related to both initial opioid prescribing characteristics and chronic opioid use after hysterectomy. ${ }^{6-11,31}$ Previous studies demonstrated that age, certain pain conditions, and psychiatric disorders are associated with both postsurgical chronic pain and chronic prescription opioid use. ${ }^{6,21,31-36}$ Covariates assessed in our analyses included age at hysterectomy, Charlson comorbidity index, hospital length of stay, smoking status, use of alcohol and illicit substances, psychiatric medication use, pre-operative pain conditions, types of hysterectomy procedure performed, US census region, type of insurance (Medicare or private insurance) and health plan (exclusive provider organization, health maintenance organization, point of service, and others including indemnity and preferred provider organization), year of surgery, and initial opioid prescribing characteristics (medication type, average daily dose, and number of days' supplied).

Statistical Analysis: Categorical variables were examined and compared using Chi-squared or Fisher exact tests. Continuous variables were compared using the student $t$ test. Statistical tests and modeling were conducted to identify risk factors for chronic opioid use among women after hysterectomy. A multivariable logistic regression model was used to estimate the association between baseline demographic and clinical variables, initial opioid prescribing characteristics, and chronic opioid use after hysterectomy among opioid-naive patients who filled at least one opioid prescription within 7 days after hysterectomy.

Sensitivity studies were conducted using multivariate logistic regression models to examine if the significant association between initial prescribing pattern and subsequent chronic opioid use were upheld with a different definition of chronic opioid use post hysterectomy or a more restricted definition for opioid naivety after adjusting for other covariates. 
A probability of type 1 error (alpha) $=0.05$ was set as the threshold of statistical significance. The trajectory models were conducted using "Proc Traj". All statistical analyses were performed using Statistical Analysis Software (SAS), version 9.4 (SAS Institute Inc, Cary, NC).

\section{Results}

Study cohort: A total of 157,934 women underwent hysterectomy between 07/01/2010 and 03/31/2015. Of these patients, $22,186(14 \%)$ patients did not meet the continuous enrollment criteria, 14,696 (9\%) had suspected cancer diagnoses or radical hysterectomy, 18,202 (12\%) didn't fill an opioid prescription within 7 days of surgery, $3,918(2 \%)$ were missing important demographic data, 48,778 (31\%) used opioids in the 6 months prior to surgery and $232(0.1 \%)$ were diagnosed with OUD within 6 months prior to hysterectomy, leaving 50,127 (32\%) patients for the analysis. A total of 12,985 (26\%) patients had an abdominal hysterectomy; 29,640 (59\%) patients had a laparoscopic or robotic assisted laparoscopic hysterectomy; and 7,502 (15\%) patients had a vaginal hysterectomy (Figure 1).

The trajectory model identified 4 distinct trajectories of prescription opioid use after hysterectomy, with total $39,677(79.15 \%)$ patients in trajectory $1,3,304(6.59 \%)$ in trajectory 2 , $6,453(12.87 \%)$ in trajectory 3 , and $693(1.38 \%)$ in trajectory 4 . (Figure 2). Among the patients in trajectory $4(n=693,1.38 \%), 57 \%, 55 \%, 57 \%, 55 \%, 48 \%$, and $41 \%$ filled an opioid prescription at 1 month, 2 months, 3 months, 4 months, 5 months, and 6 months following an initial prescription in the 7 days post-hysterectomy, respectively. Patients in trajectory 4 were classified as chronic opioid users. No patient in trajectory $1(n=39,677,79.15 \%)$ filled any opioid prescription ( $0 \%)$ from month 1 to 6 . Among patients in trajectory $2(n=3,304,6.59 \%), 0.3 \%$ filled a prescription at month 1 , which increased to $2.6 \%$ at month $2,8 \%$ at month 3 , and then followed by $10 \%$ with opioid fills for the subsequent 3 months. Thirty-five percent of patients in trajectory $3(n=6,543,12.87 \%)$ filled an opioid prescription at month 1 , which decreased to $8 \%$ 
at month 2 , and then gradually decreased to $4 \%$ at month 3 , and $3 \%$ at months 4,5 , and 6 . A description of the demographic and clinical characteristics of the patients assigned to each of these 4 trajectories is included in eTable2.

Characteristics of patients and initial prescriptions: Among the total 50,127 opioid naïve patients who filled at least one opioid prescription within 7 days after hysterectomy, 693 (1.38\%) patients were categorized as chronic opioid users and $48,434(98.62 \%)$ were categorized as non-chronic users. Baseline characteristics among chronic opioid users after hysterectomy were significantly different from non-chronic opioid users (Table 1). Compared to the women without chronic opioid use, women with chronic opioid use were older and more likely to have a history of tobacco use, and a history of alcohol use. Chronic opioid users also were also more likely to be taking psychiatric medications, including benzodiazepines, antidepressants, and stimulants. Back pain and headache syndromes were more prevalent in the chronic opioid use group. Compared to women with commercial insurance, women with Medicare insurance had higher rates of chronic opioid use. About 1.5 percent of Medicare patients who were chronic opioid users after hysterectomy were younger than 65 .

In unadjusted analyses, the average daily MME/day and the type of the initial opioid prescription were similar between women categorized as having chronic use and not having chronic opioid use. The days' supplied of the initial opioid was marginally greater among chronic users than for non-chronic users (5.12 versus 4.85 days, respectively, $p=0.02)$ (Table 1$)$. Overall, oxycodone was more frequently prescribed hydrocodone or other opioids.

\section{Predictors of chronic opioid use among all patients in the study: The results of} multivariable logistic regression analyses are shown in Table 2. A number of factors were significantly associated with chronic opioid use after hysterectomy including older age, abdominal or laparoscopic/robotic hysterectomy, Charlson comorbidity index, tobacco use, alcohol use, and prescribed psychotropic medications. Pain conditions, including headache syndromes and back pain at baseline, were also identified as significant risk factors for chronic 
opioid use after hysterectomy. Health plan type, census region, surgical setting, fibromyalgia, and other substance use disorders were not included in the final multivariable analyses due to lack of significance.

The characteristics of the initial opioid prescription, including type of opioid dispensed, days' supply, and daily dose in MME, were fitted in the model. Among initial opioid prescribing characteristics, significant predictors of chronic opioid use were initial prescription of hydrocodone (compared to oxycodone, aOR: $1.31,95 \% \mathrm{Cl}: 1.10-1.57$ ), days' supply (4-7 days vs $\leq 3$ days, aOR: $1.28,95 \% \mathrm{Cl}: 1.06-1.54 ; \geq 8$ days vs $\leq 3$ days, aOR: $1.41,95 \% \mathrm{Cl}: 1.05-1.89$ ), and daily dose in MME ( $\geq 60 \mathrm{mg} /$ day vs $\leq 40 \mathrm{mg} /$ day, aOR: $1.43,95 \% \mathrm{Cl}: 1.14-1.79)$, The Cstatistic for the fitted full model was 0.70 , indicating moderate predictability (Table 2)

The frequencies of post-surgery complications and other conditions associated with pain during the 6-month follow-up were examined in the study cohort (Table 3). Compared with nonchronic opioid users, women who developed chronic opioid use after hysterectomy were more frequently diagnosed with chronic pancreatitis, headache syndrome, back pain, fibromyalgia, and underwent additional anesthetic procedures.

\section{Sensitivity Studies}

We redefined chronic opioid use as a total duration of opioid use (total days' supply) that exceeded 90 days during 6-month follow-up period, finding that the days supplied and MME of the initial opioid prescription were associated with chronic opioid use according to this definition. An initial days supply was associated with an adjusted 1.81 fold increase in chronic use as compared with a supply of less than 3 days (95\% Cl: $1.21-2.70)$; while an initial MME/day of 60 or greater was associated with an adjusted 1.37 fold increase in chronic use $(95 \% \mathrm{Cl}: 1.00-1.87)$ as compared with an initial MME of $\leq 39.64 \mathrm{mg} / \mathrm{day}$ ). These results are similar as the results which defined chronic use using trajectory models. 
In another sensitivity analysis, we applied a more restricted definition of opioid naivety by only including patients having 12-month continuous eligibility and without any opioid dispensing or diagnosis of OUD during the 12 months prior to hysterectomy. In this analysis, the highest tertile of MME/day (exceeding $58.92 \mathrm{mg}$ ) for the initial opioid prescription remained predictive of subsequent chronic opioid use as compared with the lowest tertile of $\leq 39.64 \mathrm{mg} / \mathrm{day}$ (aOR: 1.38; $95 \% \mathrm{Cl}: 1.07-1.80)$; while the trend for days supplied also remained consistent, albeit failing to reach statistical significance. (aOR: $1.38,95 \% \mathrm{Cl}$ : 1.0-1.90 for $8+$ days' supply vs $\leq 3$ days' supply)

\section{Discussion}

Hysterectomy is the most common surgical procedure among non-pregnant women. In our study, among the 50,127 opioid naïve women who had a hysterectomy and were dispensed at least one opioid prescription within 7 days after hysterectomy, 1.38\% became chronic opioid users during the 6 months after surgery. Several demographic factors, such as age, mode of hysterectomy, tobacco use, alcohol use, and psychiatric use were associated with chronic opioid use after hysterectomy. More importantly, however, we found that characteristics of the initial opioid prescription, including type, dose, and duration affected the probability of chronic opioid use. An initial supply of 8 or more days, and an initial MME of at least $60 \mathrm{mg}$ per day were each associated with a greater than $40 \%$ increase in the risk of subsequent chronic use of opioids (Table 2). Each year in the United States, there are approximately 430,000 inpatient hysterectomy cases. ${ }^{37}$ The findings of this study suggest that lessening the duration and amount of opioids in the initial opioid prescription may decrease in the risk of chronic opioid use after hysterectomy.

Multiple demographic and clinical factors were associated with increased risk of chronic opioid use after hysterectomy, including older age, abdominal or laparoscopic/robotic hysterectomy, comorbidities, tobacco use, substance use disorders, certain pain conditions, and 
use of prescription psychiatric medications use (Table 2). Our findings were similar to other studies that showed that these factors increase the risk of chronic opioid use after major cardiac, thoracic, abdominal and pelvic procedures, or specific surgeries, including cesarean delivery, hysterectomy, hip or knee arthroplasty, spine, or bariatric surgeries. ${ }^{5-17,} 38,39$ These findings underscore the importance of considering the individual patient's pain management needs,risk factors for opioid misuse at the time of opioid prescribing after hysterectomy and other surgical procedures, and importance of medical care, monitoring and follow-up postoperatively .

The literature describing the relationship between the initial opioid prescribing characteristics and the risk of chronic opioid use after hysterectomy among opioid naïve patients is limited. Prior observational studies have produced inconclusive results, which may be attributed to the study sample (inclusion of patients with minor surgical procedures) or small study sample size. ${ }^{11,38}$ Our results showed that initial opioid prescribing characteristics, including opioid type, daily dose, and duration of days' supply were associated with chronic opioid use in the 6 months following hysterectomy among opioid naïve patients. The odds of chronic opioid use following initiation after hysterectomy were $31 \%$ higher among patients starting hydrocodone compared to patients who began therapy with oxycodone even though prior research has demonstrated that oxycodone is associated with risk of addiction, morbidity and mortality. ${ }^{40-42}$ One explanation for this finding is that for many years hydrocodone was the most commonly prescribed opioid medication in the United States, in part because it was a schedule III drug during the study period and was considered to be lesser risk than schedule II opioids such as oxycodone, therefore, hydrocodone may have been prescribed more liberally, especially to patients with a higher tendency of opioid overuse, which is a possible confounding by contraindication. In August 21, 2014, the U.S. Drug Enforcement Administration (DEA) issued stricter prescribing requirements and moved hydrocodone-containing medications from a Schedule III to a Schedule II controlled substance..$^{43}$ Notably, the initial opioid prescribing 
characteristics, such as longer days' supply and higher daily morphine milligram equivalent dose, that were associated with post-operative chronic opioid use after hysterectomy, are also risk factors for opioid misuse and opioid-related mortality. ${ }^{44-47}$ By identifying modifiable risk factors for chronic opioid use after hysterectomy, such as pre-operative opioid prescribing, abdominal or laparoscopic/robotic hysterectomy, and characteristics of opioid prescribing after surgery, we may be able to develop strategies and interventions to decrease the likelihood of chronic opioid use.

There is no standard definition for chronic opioid use after surgery. We selected the trajectory modeling approach because of its advantages over other methods for describing longitudinal trajectories and identifying patients who used opioids consistently over a longer duration. ${ }^{29,30}$ Traditional medication adherence measures, such as the proportion of days covered or the number of months of continuous medication use, may not distinguish between consistent users in follow-up periods or between patients who discontinue medication completely versus those who simply have a gap in use. The trajectory models repeatedly assess medication use throughout the entire follow-up period and summarize long-term medication adherence accounting for the time-varying nature of adherence. ${ }^{29} \mathrm{We}$ found that $1.38 \%$ of hysterectomy patients became chronic opioid users in the 6 months after surgery. Using Optum data from January 01, 2011 to December 31, 2014, Swenson et al. reported 0.5\% (122 out of 24,331 ) of women who had a hysterectomy had new persistent opioid use following hysterectomy. ${ }^{5}$ In Swenson's study, persistent opioid use in the 6 months post-hysterectomy was defined as a minimum of 2 opioid prescriptions in the 6 months post-surgery (one between 15-90 days and one at least $91-180$ days) and top quartile use (amount and duration). ${ }^{5}$ The difference in incidence of chronic use/new persistent use between these studies is attributable to the use of different definitions for chronic opioid use.

Our findings suggest that prescribing lower doses for shorter days' supply may reduce the risk of subsequent chronic opioid use, and is consistent with the strategies published in 
guidelines advocated by multiple federal and state agencies, professional societies, and advocacy groups. Although specific guidelines differ in exact wording and specific recommendations, prescribing the lowest dose for the shortest amount of time and screening for substance use and opioid dependence are clearly defined themes across multiple guidelines. ${ }^{48-}$

50 However, practice change solely on the basis of guidelines can be slow, and current evidence suggests that physicians are prescribing excess opioids to patients to control acute pain postsurgery at hospital discharge. Studies from the surgical fields of urology, orthopedics, and obstetrics and gynecology have shown that physicians prescribe more than twice the amount of opioid medication patients actually consume post-discharge which adds up to millions of excess unused opioid tablets available for diversion and abuse..$^{51-54} \mathrm{~A}$ recent study showed that among obstetricians and gynecologists, only $62 \%$ reported tailoring prescriptions to the individual patient and only $22 \%$ reported they performed an opioid dependence screen prior to prescribing. ${ }^{55}$ Improving adherence to best practices for opioid prescribing, including tailoring prescriptions to the individual patient's pain management needs and risks of future opioid misuse and abuse, could play a major role in reducing the magnitude of the opioid epidemic.

\section{Limitations}

First, the study subjects included in this study are commercially insured in the United States with $9 \%$ covered by Medicare Advantage (Table1). The demographic characteristics, clinical conditions, and opioid preoperative use patterns may differ from uninsured, Medicaid or Medicare covered populations. Thus, the study results might not be generalizable to patients enrolled in Medicaid or Medicare.

Our study is subject to several limitations due to the nature of observational studies using claims data. Some important medical indications, such as severity of pain, which may be related to the hysterectomy procedure or underlying conditions leading to hysterectomy, were 
not captured in the data. Other unmeasured confounding factors included social and economic factors during follow up. The low prevalence of OUDs or methadone use excluded in figure 1, and other substance use disorders, tobacco use, and alcohol use presented in table 1 might be due to poor sensitivity of ICD-9 diagnosis codes or CPT codes. However, the impact may be non-differential if the unmeasured variables are similarly distributed in two comparison groups.

Opioid analgesics were assessed using pharmacy claims, which only captured opioid medications legally obtained and filled at outpatient pharmacies. Patients not filling the initial opioid prescription post-hysterectomy may be attributed to either not using opioids for postsurgical pain or to receiving their opioid prescription from an inpatient pharmacy. The actual patterns of opioid use (actual consumption) was not measured and, based on prior studies, differs substantially from the amount of opioid medication prescribed. Furthermore, the result that hydrocodone has a higher risk of chronic use after hysterectomy compared to oxycodone could be due to a possible confounding by contraindication.

The outcome assessed in this study was chronic opioid use during the first 6 months after hysterectomy. Although chronic opioid use within 6 months has been associated with opioid misuse and opioid-related death, it was not possible to differentiate whether patients persistently used opioids for 6 months as a treatment for pain control or because they became dependent upon opioids after their post-surgical pain had abated. ${ }^{56}$ Additionally, it was not possible to determine if the women were continuing to receive their opioid for the pain related to their hysterectomy or for other pain; It is possible that the reason for filling opioid prescriptions in the 6 months following hysterectomy was not for personal use but for diversion. ${ }^{57}$ Our findings that chronic opioid use post hysterectomy was significantly associated with initial opioid prescribing could be due to complex relationships between chronic pain postsurgery, acute pain post-surgery, and chronic pain pre-surgery. 


\section{Conclusions}

In this study, approximately 3 in 200 opioid naïve women appear to become chronic opioid users after hysterectomy. Besides commonly recognized demographic and clinical risk factors, chronic opioid use after hysterectomy was associated with initial opioid prescribing characteristics, such as longer days' supply and higher daily opioid dose. Although prescription opioid medications provide effective analgesia after surgery, they must be used with caution given the potential risk for subsequent chronic opioid use that is associated with opioid misuse and overdose-related mortality. Our findings support the need to manage post-surgical pain with the least amount of opioid medication possible to effectively control a patient's symptoms, and close clinical post-op follow-up for those patients who prescribed opioids. 


\section{References}

1. The U.S. Department of Health and Human Services Office on Women's Health. White Paper: Opioid Use, Misuse, and Overdose in Women.

https://www.womenshealth.gov/files/documents/white-paper-opioid-508.pdf. Accessed 01/18/17.

2. McCarthy M. Opioid overdose deaths rose fivefold among US women in 10 years. BMJ 2013;347:f4415.

3. Chou R, Ballantyne JC, Fanciullo GJ, Fine PG, Miaskowski C. Research Gaps on Use of Opioids for Chronic Noncancer Pain: Findings From a Review of the Evidence for an American Pain Society and American Academy of Pain Medicine Clinical Practice Guideline. The Journal of Pain, Vol 10, No 2 (February), 2009: pp 147-159

4. An analysis of the impact of opioid overprescribing in America. United States for nondependence. The role of opioids in treating postsurgical pain. Pg 9-13. https://www.planagainstpain.com/wpcontent/uploads/2017/09/PlanAgainstPain USND.pdf. The Plan Against Pain. September 26, 2017. Accessed December 20, 2018.

5. Swenson CW, Kamdar NS, Seiler K, Morgan DM, Lin P, As-Sanie S. Definition development and prevalence of new persistent opioid use following hysterectomy. Am J Obstet Gynecol. 2018 Nov;219(5):486.e1-486.e7.

6. Bateman BT, Franklin JM, Bykov K, et al. Persistent opioid use following cesarean delivery: patterns and predictors among opioid-naïve women. Am J Obstet Gynecol. 2016; S0002-9378(16)00478-6.

7. Hansen CA, Inacio MC, Pratt NL, Roughead EE, Graves SE. Chronic Use of Opioids Before and After Total Knee Arthroplasty: A Retrospective Cohort Study. J Arthroplasty. 2016 Oct 4. 
8. Clarke H, Soneji N, Ko DT, Yun L, Wijeysundera DN. Rates and risk factors for prolonged opioid use after major surgery: population based cohort study. BMJ. 2014;348:g1251.

9. Hetmann F, Kongsgaard UE, Sandvik L, Schou-Bredal I. Prevalence and predictors of persistent post-surgical pain 12 months after thoracotomy. Acta Anaesthesiol Scand. 2015;59(6):740-748.

10. Sun EC, Darnall BD, Baker LC, Mackey S. Incidence of and Risk Factors for chronic opioid use among opioid-naive patients in the postoperative period. JAMA Intern Med. 2016 Sep 1;176(9):1286-93.

11. Raebel MA, Newcomer SR, Reifler LM, Boudreau D, Elliott TE, DeBar L, Ahmed A, Pawloski PA, Fisher D, Donahoo WT, Bayliss EA. Chronic use of opioid medications before and after bariatric surgery. JAMA. 2013 Oct 2;310(13):1369-76.

12. Alam A, Gomes T, Zheng H, Mamdani MM, Juurlink DN, Bell CM. Long-term analgesic use after low-risk surgery: a retrospective cohort study. Arch Intern Med. 2012;172(5):425-430.

13. Calcaterra SL, Scarbro S, Hull ML, Forber AD, Binswanger IA, Colborn KL. Prediction of Future Chronic Opioid Use Among Hospitalized Patients. J Gen Intern Med. 2018 Jun;33(6):898-905. doi: 10.1007/s11606-018-4335-8. Epub 2018 Feb 5.

14. Rao AG, Chan PH, Prentice HA, Paxton EW, Navarro RA, Dillon MT, Singh A. Risk factors for postoperative opioid use after elective shoulder arthroplasty. J Shoulder Elbow Surg. 2018 Jun 8.

15. Jafari A, Shen SA, Bracken DJ, Pang J, DeConde AS. Incidence and predictive factors for additional opioid prescription after endoscopic sinus surgery. Int Forum Allergy Rhinol. 2018 May 31. 
16. Namba RS, Singh A, Paxton EW, Inacio MCS. Patient Factors Associated With Prolonged Postoperative Opioid Use After Total Knee Arthroplasty. J Arthroplasty. 2018 Apr 9.

17. Pugely AJ, Bedard NA, Kalakoti P, Hendrickson NR, Shillingford JN, Laratta JL, Saifi C, Lehman RA, Riew KD. Opioid use following cervical spine surgery: trends and factors associated with long-term use. Spine J. 2018 Apr 10. pii: S1529-9430(18)30129-3.

18. Dunn LK, Yerra S, Fang S, Hanak MF, Leibowitz MK, Tsang S, Durieux ME, Nemergut EC, Naik BI. Incidence and Risk Factors for Chronic Postoperative Opioid Use After Major Spine Surgery: A Cross-Sectional Study With Longitudinal Outcome. Anesth Analg. $2018 \mathrm{Jul} ; 127(1): 247-254$.

19. Merrill RM. Hysterectomy surveillance in the United States, 1997 through 2005. Med Sci Monit. Jan; 2008 14(1):CR24-31.

20. Darnall B, Li H. Hysterectomy and predictors for opioid prescription in a chronic pain clinic sample. Pain Med. 2011 Feb;12(2):196-203.

21. Merrill RM, Layman AB, Oderda G, Asche C. Risk estimates of hysterectomy and selected conditions commonly treated with hysterectomy. Ann Epidemiol. Mar; 2008 18(3):253-260.

22. Brandsborg B, Nikolajsen L, Hansen CT, Kehlet H, Jensen TS. Risk factors for chronic pain after hysterectomy: a nationwide questionnaire and database study. Anesthesiology. May; 2007 106(5): 1003-1012.

23. Optum. Retrospective database analysis. Optum Inc. 2013. Available at: https://www.optum.com/content/dam/optum/resources/productSheets/RetrospectiveDatabase-Analysis.pdf. Accessed 01/28/2017.

24. Keshavarz H, Hillis SD, Kieke BA. Hysterectomy surveillance-United States, 19941999. MMWR. 2002;1(SS-5):1-8. 
25. Chastek B, Harley C, Kallich J, Newcomer L, Paoli CJ, Teitelbaum AH. Health care costs for patients with cancer at the end of life. J Oncol Pract. 2012 Nov;8(6):75s-80s.

26. Jones BL, Nagin DS. Advances in group-based trajectory modeling and an SAS procedure for estimating them. Socio Meth Res 2007; 35(4): 542-571.

27. Schwarz G. Estimating the dimension of a model. Ann Stat 1978; 6(2): 461-464.

28. Nagin DS. Analyzing developmental trajectories: a semiparametric, group-based approach. Psychol Methods 1999;4:139.

29. Franklin JM, Shrank WH, Pakes J, et al. Group-based trajectory models: a new approach to classifying and predicting long-term medication adherence. Med Care 2013;51:789-96.

30. Jones BL, Nagin DS. Advances in group-based trajectory modeling and an SAS procedure for estimating them. Socio Meth Res 2007; 35(4): 542-571.

31. Turk DC, Okifuji A. What factors affect physicians' decisions to prescribe opioids for chronic noncancer pain patients? Clin J Pain. Dec; 1997 13(4):330-336.

32. Sullivan MD, Edlund MJ, Zhang L, Unutzer J, Wells KB. Association between mental health disorders, problem drug use, and regular prescription opioid use. Arch Intern Med. Oct 23; 2006 166(19):2087-2093.

33. Hooten WM, Townsend CO, Bruce BK, et al. Effects of smoking status on immediate treatment outcomes of multidisciplinary pain rehabilitation. Pain Med. Mar; 2009 $10(2): 347-355$.

34. Rudd RA, Aleshire N, Zibbell JE, Gladden MR, Increases in Drug and Opioid Overdose Deaths - United States, 2000-2014.

http://www.cdc.gov/mmwr/preview/mmwrhtml/mm6450a3.htm. Accessed 01/26/18.

35. Brandborg B, Dueholm M, Nikolajsen L, Kehlet H, Jensen TS. A prospective study of risk factors for pain persisting 4 months after hysterectomy. Clin J Pain. 2009 May;25(4):263-8. 
36. Calderon M, Castorena G, Pasic E. Postoperative pain management after hysterectomy - a simple approach. http://cdn.intechopen.com/pdfs/35360/InTech-

\section{Postoperative pain management after hysterectomy a simple approach.pdf.}

\section{Accessed 01/24/17.}

37. Wright JD, Herzog TJ, Tsui J, et al. Nationwide trends in the performance of inpatient hysterectomy in the United States. Obstet Gynecol. 2013;122(2 Pt 1):233-41.

38. Kim SC, Choudhry N, Franklin JM, Bykov K, Eikermann M, Lii J, Fischer MA, Bateman BT. Patterns and predictors of persistent opioid use following hip or knee arthroplasty. Osteoarthritis Cartilage. 2017 Sep;25(9):1399-1406. doi: 10.1016/j.joca.2017.04.002. Epub 2017 Apr 19.

39. Soneji N, Clarke HA, Ko DT, Wijeysundera DN. Risks of developing persistent opioid use after major surgery. JAMA Surg. 2016 Nov 1;151(11):1083-1084. doi:

10.1001/jamasurg.2016.1681

40. Solomon DH, Rassen JA, Glynn RJ, et al. The comparative safety of opioids for nonmalignant pain in older adults. Arch Intern Med. 2010;170(22):1979-1986.

41. Dhalla IA, Mamdani MM, Sivilotti ML, Kopp A, Qureshi O, Juurlink DN. Prescribing of opioid analgesics and related mortality before and after the introduction of long-acting oxycodone. CMAJ. 2009;181(12):891-896.

42. Madsen AM, Stark LM, Has P, Emerson JB, Schulkin J, Matteson KA. Opioid Knowledge and Prescribing Practices Among Obstetrician-Gynecologists. Obstet Gynecol. 2018 Jan;131(1):150-157.

43. Coleman JJ. Rescheduling Hydrocodone Combination Products: Addressing the Abuse of America's Favorite Opioid. ASAM.

https://www.asam.org/resources/publications/magazine/read/article/2015/04/10/resched uling-hydrocodone-combination-products-addressing-the-abuse-of-america-s-favoriteopioid. Accessed July 12, 2018. 
44. Bohnert AS, Valenstein M, Bair MJ, et al. Association between opioid prescribing patterns and opioid overdose-related deaths. Jama. Apr 6 2011;305(13):1315-1321.

45. Jones JD, Mogali S, Comer SD. Polydrug abuse: A review of opioid and benzodiazepine combination use. Drug and alcohol dependence. 9/1/ 2012;125(1-2):818.

46. Paulozzi LJ, Zhang K, Jones CM, Mack KA. Risk of adverse health outcomes with increasing duration and regularity of opioid therapy. Journal of the American Board of Family Medicine : JABFM. May-Jun 2014;27(3):329-338.

47. Gwira Baumblatt JA, Wiedeman C, Dunn JR, Schaffner W, Paulozzi LJ, Jones TF. High-risk use by patients prescribed opioids for pain and its role in overdose deaths. JAMA internal medicine. May 2014;174(5):796-801.

48. Washington State Agency Medical Directors' Group. Interagency Guideline on Prescribing Opioids for Pain 2015. Available from: http://www.agencymeddirectors.wa.gov/Files/2015AMDGOpioidGuideline.pdf.

49. Alexander GC, Frattaroli S, Gielen AC, eds. The Prescription Opioid Epidemic: An Evidence-Based Approach. Johns Hopkins Bloomberg School of Public Health, Baltimore, Maryland: 2015. Available from: https://www.drugabuse.gov/nidamedmedical-health-professionals/opioid-pain-management-cmes/unaccredited-module-1safe-prescribing-pain. Accessed: July 24, 2018.

50. Dowell D, Haegerich TM, Chou R. CDC Guideline for Prescribing Opioids for Chronic Pain - United States, 2016. MMWR Recommendations and Reports: Morbidity and Mortality Weekly Report. 2016;65(1):1-49. PMID: 26987082.

51. Bartels K, Mayes LM, Dingmann C, Bullard KJ, Hopfer CJ, Binswanger IA. Opioid use and storage patterns by patients after hospital discharge following surgery. PloS one. 2016;11(1):e0147972. PMID: 26824844; PMCID: PMC4732746. 
52. Bates C, Laciak R, Southwick A, Bishoff J. Overprescription of postoperative narcotics: a look at postoperative pain medication delivery, consumption and disposal in urological practice. J Urol. 2011;185(2):551-5. PMID: 21168869.

53. Chapman T, Kim N, Maltenfort M, Ilyas AM. Prospective evaluation of opioid consumption following carpal tunnel release surgery. Hand. 2017;12(1):39-42. PMID: 28082841; PMCID: PMC5207284.

54. Kim N, Matzon JL, Abboudi J, Jones C, Kirkpatrick W, Leinberry CF, et al. A prospective evaluation of opioid utilization after upper-extremity surgical procedures: identifying consumption patterns and determining prescribing guidelines. JBJS American. 2016;98(20):e89. PMID: 27869630.

55. Madsen AM, Stark LM, Has P, Emerson JB, Schulkin J, Matteson KA. Opioid Knowledge and Prescribing Practices Among Obstetrician-Gynecologists. Obstet Gynecol. 2018 Jan;131(1):150-157. PubMed PMID:29215508.

56. Paulozzi LJ, Strickler GK, Kreiner PW, Koris CM, Centers for Disease C, Prevention. Controlled Substance Prescribing Patterns--Prescription Behavior Surveillance System, Eight States, 2013. Morbidity and mortality weekly report. Surveillance summaries. Oct 16 2015;64(9):1-14.

57. Neuman MD, Bateman BT, Wunsch H, Postoperative pain management and opioids 2 Inappropriate opioid prescription after surgery. The Lancet. Vol 393, Issue 10180, 13-19 April 2019, Pg 1547-1557. 
Figure 1. Flow Chart of the Study Population.

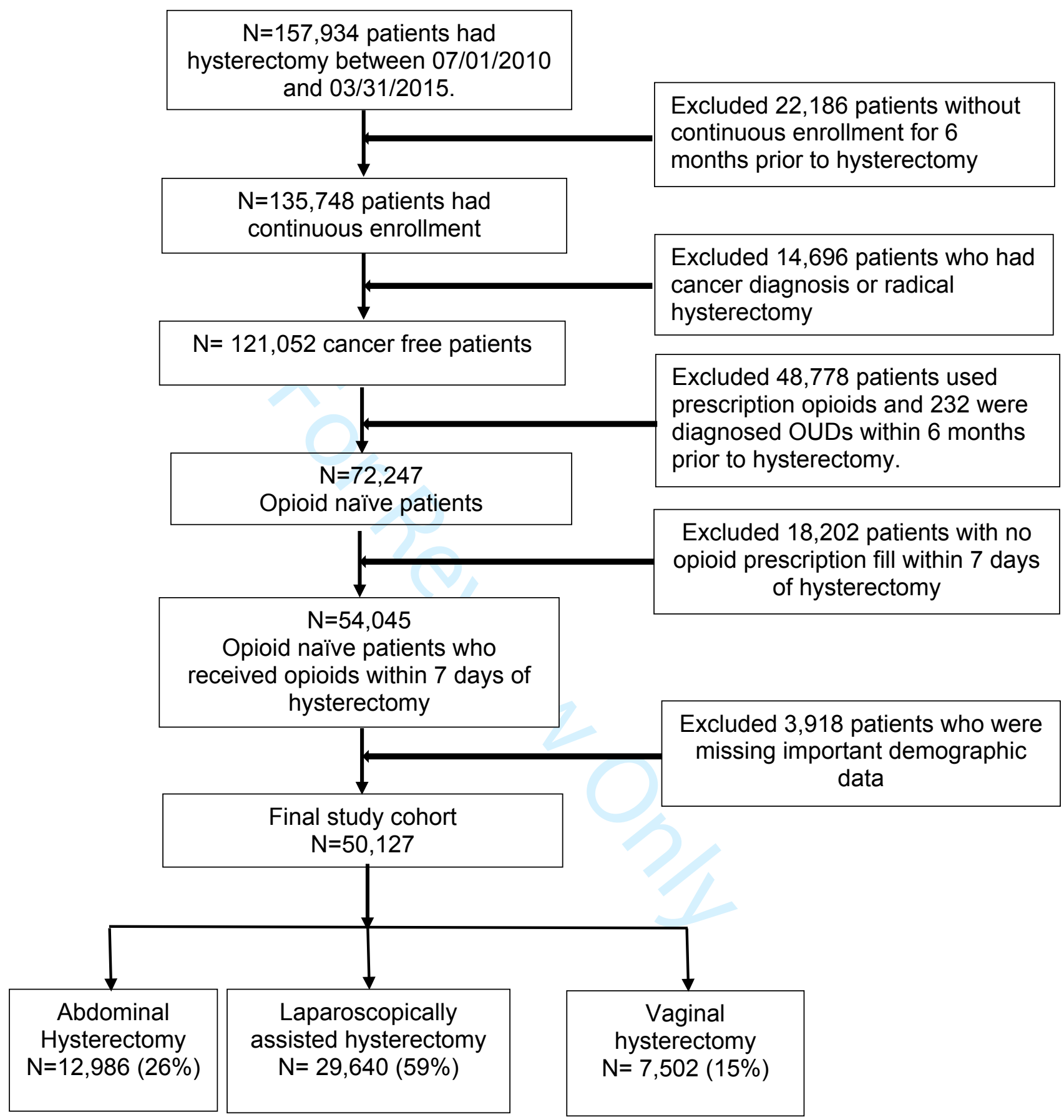


Figure 2. Trajectories of filling opioid prescriptions in 6 months post hysterectomy. The solid or dashed lines represent the distinct estimated opioid prescription filling trajectories. The dot symbols represent the mean estimated proportion in each trajectory group dispensed an opioid prescription at each month. These indicate the mean estimated probability of filling an opioid prescription for patients in each group at each time point. Overall counts and percentages of patients in each group are presented in the underneath table. Patients in trajectory 4 were classified as opioid chronic users. Patients in trajectories 1, 2, 3 were classified as opioid nonchronic users. The x-axis indicates each 30-day interval during the 6-month follow-up period, while the $y$-axis indicates the estimated proportion of patients filled a prescription opioid within each 30-day interval.

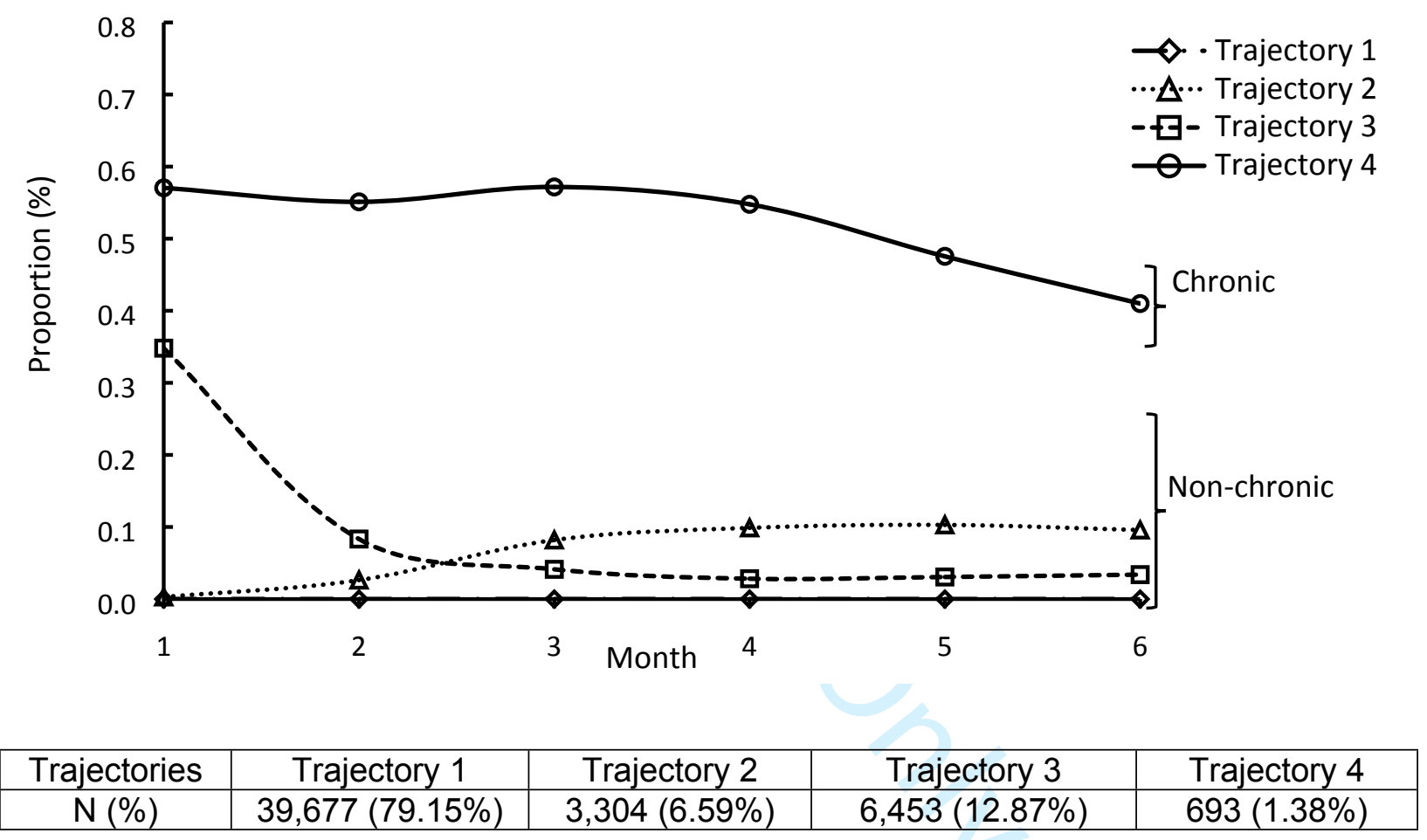


Table 1. Comparison of baseline patient characteristics among opioid-naïve patients who received prescription opioids within 7 days after hysterectomy, $\mathrm{N}=50,127$

\begin{tabular}{|c|c|c|c|}
\hline Patient characteristics & $\begin{array}{l}\text { Non-chronic } \\
\text { Users }^{\dagger} \\
(\mathrm{N}=48,434)\end{array}$ & $\begin{array}{l}\text { Chronic Opioid Users } \\
\qquad(\mathrm{N}=693)\end{array}$ & P-value \\
\hline Charlson Comorbidity Index, mean (95\% Cl) & $1.60(1.57,1.64)$ & $1.74(1.45,2.02)$ & 0.3479 \\
\hline Length of stay in days, mean $(95 \% \mathrm{Cl})$ & $2.32(2.30,2.35)$ & $3.79(3.39,4.19)$ & $<0.0001$ \\
\hline Age, years, mean $(95 \% \mathrm{Cl})$ & $48.1(48.0,48.2)$ & $49.8(48.9,50.7)$ & 0.0004 \\
\hline $\begin{array}{l}\text { Age at hysterectomy, years } \\
\quad<40 \\
40-49 \\
50+\end{array}$ & $\begin{array}{c}9,100(18.41) \\
22,538(45.59) \\
17,796(36.00)\end{array}$ & $\begin{array}{l}126(18.18) \\
255(36.80) \\
312(45.02)\end{array}$ & $<.0001$ \\
\hline $\begin{array}{l}\text { Substance use and abuse, N (\%) } \\
\text { Tobacco } \\
\text { Alcohol } \\
\text { Other substance use disorders }\end{array}$ & $\begin{array}{c}2,637(5.33) \\
177(0.36) \\
55(0.11)\end{array}$ & $\begin{array}{l}60(8.66) \\
9(1.30) \\
4(0.58)\end{array}$ & $\begin{array}{l}0.0001 \\
<.0001 \\
0.0004\end{array}$ \\
\hline $\begin{array}{l}\text { Psychiatric medications, N (\%) } \\
\text { Benzodiazepines } \\
\text { Antidepressants } \\
\text { Stimulants }\end{array}$ & $\begin{array}{c}4,201(8.50) \\
9,984(20.20) \\
767(1.55)\end{array}$ & $\begin{array}{c}125(18.04) \\
233(33.62) \\
25(3.61)\end{array}$ & $\begin{array}{l}<.0001 \\
<.0001 \\
<.0001\end{array}$ \\
\hline $\begin{array}{l}\text { Pain conditions, } \mathrm{N}(\%) \\
\text { Fibromyalgia } \\
\text { Headache syndromes } \\
\text { Back pain }\end{array}$ & $\begin{array}{l}1,530(3.10) \\
4,019(8.13) \\
4,659(9.42)\end{array}$ & $\begin{array}{c}30(4.33) \\
82(11.83) \\
110(15.87)\end{array}$ & $\begin{array}{l}0.0632 \\
0.0004 \\
<.0001\end{array}$ \\
\hline $\begin{array}{l}\text { Type of hysterectomy, } \mathrm{N}(\%) \\
\text { Abdominal hysterectomy } \\
\text { Vaginal hysterectomy } \\
\text { Laparoscopic/Robotic }\end{array}$ & $\begin{array}{c}12,757(25.81) \\
7,428(15.03 \\
29,249(59.17)\end{array}$ & $\begin{array}{l}228(32.90) \\
74(10.68) \\
391(56.42)\end{array}$ & $<.0001$ \\
\hline $\begin{array}{l}\text { Surgery setting, N (\%) } \\
\text { Inpatient } \\
\text { Outpatient }\end{array}$ & $\begin{array}{l}19,805(40.06) \\
29,629(59.94)\end{array}$ & $\begin{array}{l}352(50.79) \\
341(49.21)\end{array}$ & $<.0001$ \\
\hline $\begin{array}{l}\text { US census region, } \mathrm{N}(\%) \\
\text { Midwest } \\
\text { Northeast } \\
\text { South } \\
\text { West }\end{array}$ & $\begin{array}{c}13,705(27.72) \\
5,075(10.27) \\
23,642(47.83) \\
7,012(14.18)\end{array}$ & $\begin{array}{c}186(26.84) \\
66(9.52) \\
332(47.91) \\
109(15.73)\end{array}$ & 0.6351 \\
\hline $\begin{array}{l}\text { Insurance type, N (\%) } \\
\text { Commercial } \\
\text { Medicare }\end{array}$ & $\begin{array}{c}45,107(91.25) \\
4,327(8.75)\end{array}$ & $\begin{array}{l}586(84.56) \\
107(15.44)\end{array}$ & $<.0001$ \\
\hline $\begin{array}{l}\text { Type of health plan, } \mathrm{N}(\%) \\
\text { Exclusive provider organization } \\
\text { Health maintenance organization } \\
\text { Point of service } \\
\text { Others }\end{array}$ & $\begin{array}{c}5,990(12.12) \\
4,913(9.94) \\
34,004(68.79) \\
4,527(9.16)\end{array}$ & $\begin{array}{c}70(10.10) \\
89(12.84) \\
449(64.79) \\
85(12.27)\end{array}$ & 0.0008 \\
\hline $\begin{array}{c}\text { Year of hysterectomy } \\
2010-2013 \\
2014-2015\end{array}$ & $\begin{array}{l}21,949(44.40) \\
27,485(55.60)\end{array}$ & $\begin{array}{l}287(41.41) \\
406(58.59)\end{array}$ & 0.1161 \\
\hline
\end{tabular}


2

Initial Opioid Prescription Characteristics

\begin{tabular}{|l|c|c|c|}
\hline Days' supply, days & $4.85(4.82,4.87)$ & $5.12(4.89,5.34)$ & 0.0205 \\
\hline Average daily MME, mg/day & $54.7(54.3,55.0)$ & $58.4(54.6,62.2)$ & 0.0571 \\
\hline Type of Opioid, N(\%) & & & \\
Hydrocodone & $17,555(35.51)$ & $266(38.38)$ & 0.0553 \\
Oxycodone & $27,434(55.50)$ & $354(51.08)$ & \\
Other & $4,445(8.99)$ & $73(10.53)$ & \\
\hline Tertile MME, mg/day & & & \\
$<=40$ & $16,154(32.68)$ & $219(31.60)$ & 0.2425 \\
$40.5-58.9$ & $16,718(33.82)$ & $221(31.89)$ & \\
$60.0+$ & $16,562(33.50)$ & $253(36.51)$ & \\
\hline Days' supply, days & & & \\
$1-3$ & $15,667(31.69)$ & $193(27.85)$ & 0.0566 \\
$4-7$ & $29,098(58.86)$ & $423(61.04)$ & \\
$8+$ & $4,669(9.44)$ & $77(11.11)$ & \\
\hline
\end{tabular}

Abbreviation: MME=Morphine Milligram Equivalent

${ }^{\dagger}$ Non-chronic users refer to patients in trajectory 1, 2, and 3 in Figure 2.

${ }^{\ddagger}$ Chronic Opioid users refer to patients in trajectory 4 in Figure 2.

${ }^{\S}$ Other substance use includes cocaine, marijuana, anxiolytic, stimulant, hallucinogenic drugs, or abuse of unspecified drugs/medications.

"Others include Indemnity and Preferred provider organization. 
Table 2. Baseline and initial opioid prescription characteristics that predict chronic opioid use following hysterectomy among opioid naive patients, 2010-2015; $\mathrm{N}=50,127$

\begin{tabular}{|c|c|c|}
\hline Characteristics & Unadjusted OR (95\% Cl) & Adjusted $\mathrm{OR}^{+}(95 \% \mathrm{Cl})$ \\
\hline $\begin{array}{l}\text { Age group, years } \\
\quad 40-49 \\
\quad<40 \\
\geq 50\end{array}$ & $\begin{array}{c}\text { Ref. } \\
1.22(0.99,1.52) \\
1.55(1.31,1.83) \\
\end{array}$ & $\begin{array}{c}\text { Ref. } \\
1.20(0.97,1.49) \\
1.37(1.141 .65) \\
\end{array}$ \\
\hline $\begin{array}{l}\text { Insurance type } \\
\text { Commercial } \\
\text { Medicare }\end{array}$ & $\begin{array}{c}\text { Ref. } \\
1.90(1.55,2.35)\end{array}$ & $\begin{array}{c}\text { Ref. } \\
1.98(1.37,2.87\end{array}$ \\
\hline $\begin{array}{l}\text { Type of health plan, } \mathrm{N}(\%) \\
\text { Exclusive provider organization } \\
\text { Health maintenance organization } \\
\text { Point of service } \\
\text { Others }{ }^{\ddagger}\end{array}$ & $\begin{array}{c}\text { Ref. } \\
1.55(1.13,2.13) \\
1.13(0.88,1.46) \\
1.61(1.17,2.21)\end{array}$ & $\begin{array}{c}\text { Ref. } \\
1.19(0.84,1.68) \\
1.10(0.85,1.42) \\
0.75(0.48,1.18)\end{array}$ \\
\hline $\begin{array}{l}\text { Type of hysterectomy } \\
\text { Vaginal hysterectomy } \\
\text { Laparoscopic/Robotic } \\
\text { Abdominal hysterectomy }\end{array}$ & $\begin{array}{c}\text { Ref. } \\
1.79(1.38,2.34) \\
1.34(1.05,1.72)\end{array}$ & $\begin{array}{c}\text { Ref. } \\
1.52(1.16,2.00) \\
1.40(1.09,1.80)\end{array}$ \\
\hline Year (2010-2013 vs 2014-2015) & $1.13(0.97,1.32)$ & $1.24(1.06,1.46)$ \\
\hline Length of stay (days) & $1.14(1.11,1.16)$ & $1.12(1.10,1.15)$ \\
\hline Charlson comorbidity index & $1.18(1.06,1.31)$ & $1.16(1.04,1.30)$ \\
\hline $\begin{array}{l}\text { Pain conditions } \\
\text { Headache (Yes vs. No) } \\
\text { Back pain (Yes vs. No) }\end{array}$ & $\begin{array}{l}1.52(1.20,1.91) \\
1.81(1.48,2.23) \\
\end{array}$ & $\begin{array}{l}1.33(1.04,1.69) \\
1.57(1.27,1.94)\end{array}$ \\
\hline $\begin{array}{c}\text { Substance use } \\
\text { Alcohol } \\
\text { Tobacco }\end{array}$ & $\begin{array}{l}3.66(1.87,7.18) \\
1.68(1.29,2.20)\end{array}$ & $\begin{array}{l}1.84(0.91,3.74) \\
1.47(1.12,1.93)\end{array}$ \\
\hline $\begin{array}{l}\text { Psychiatric medications } \\
\text { Antidepressants } \\
\text { Benzodiazepines } \\
\text { Psychostimulants }\end{array}$ & $\begin{array}{l}2.00(1.71,2.35) \\
2.37(1.95,2.88) \\
2.38(1.58,3.56)\end{array}$ & $\begin{array}{l}1.71(1.45,2.03) \\
1.89(1.54,2.33) \\
1.84(1.21,2.80)\end{array}$ \\
\hline Initia & escription Characteristic & \\
\hline $\begin{array}{l}\text { Type of opioid } \\
\text { Oxycodone } \\
\text { Hydrocodone } \\
\text { Other } \\
\end{array}$ & $\begin{array}{c}\text { Ref. } \\
1.17(1.00,1.38) \\
1.27(0.99,1.64) \\
\end{array}$ & $\begin{array}{c}\text { Ref. } \\
1.31(1.10,1.57) \\
1.26(0.96,1.64) \\
\end{array}$ \\
\hline $\begin{array}{l}\text { Days' supply, days } \\
\quad \leq 3 \\
4-7 \\
\geq 8\end{array}$ & $\begin{array}{c}\text { Ref. } \\
1.18(0.99,1.40) \\
1.34(1.03,1.75)\end{array}$ & $\begin{array}{c}\text { Ref. } \\
1.28(1.06,1.54) \\
1.41(1.05,1.89)\end{array}$ \\
\hline $\begin{array}{l}\text { Tertile MME, mg/day } \\
\quad<40 \\
\quad 40-58.9 \\
\quad \geq 60.0\end{array}$ & $\begin{array}{c}\text { Ref. } \\
0.98(0.81,1.18) \\
1.13(0.94,1.35)\end{array}$ & $\begin{array}{c}\text { Ref. } \\
1.14(0.93,1.40) \\
1.43(1.14,1.79)\end{array}$ \\
\hline
\end{tabular}

Abbreviation: $\mathrm{MME}=$ Morphine Milligram Equivalent

Adjusted model: $c$-statistic $=0.70 ;$ Hosmer-Lemeshow Goodness-of-fit Test: $X^{2}=19.30, d f=8, p=0.0133$ 
${ }^{\dagger}$ Adjusted for baseline patient demographic and clinical characteristics listed in this table. ${ }^{\ddagger}$ Others include Indemnity and Preferred provider organization. 
Table 3. Distribution of possible indications for chronic opioid use during the 6-month follow-up period after hysterectomy: shown as $\mathrm{N}(\%)$.

\begin{tabular}{|l|c|c|c|c|}
\hline Possible indications & $\begin{array}{c}\text { All patients in } \\
\text { cohort } \\
\text { (N= 50,127) }\end{array}$ & $\begin{array}{c}\text { Non-chronic } \\
\text { Users } \\
\mathbf{( N = 4 9 , 4 3 4 )}\end{array}$ & $\begin{array}{c}\text { Chronic Opioid } \\
\text { Users } \\
(\mathbf{N}=\mathbf{6 9 3})\end{array}$ & P-value \\
\hline Chronic pancreatitis & $70(0.14)$ & $63(0.13)$ & $7(1.01)$ & $<.0001$ \\
Headache syndromes & $3,284(6.55)$ & $3,196(6.47)$ & $88(12.70)$ & $<.0001$ \\
Back pain & $4,477(8.93)$ & $4,287(8.67)$ & $190(27.42)$ & $<.0001$ \\
Fibromyalgia & $1,470(2.93)$ & $1,407(2.85)$ & $63(9.09)$ & $<.0001$ \\
Additional anesthetic & & & & \\
procedures $^{+}$ & & & & \\
0 & $46,132(92.03)$ & $45,768(92.58)$ & $364(52.53)$ & \\
1 & $3,310(6.60)$ & $3,099(6.27)$ & $211(30.45)$ & \\
2 & $525(1.05)$ & $459(0.93)$ & $66(9.52)$ & \\
$\geq 3$ & $160(0.32)$ & $108(0.22)$ & $52(7.50)$ & \\
\hline
\end{tabular}

${ }^{\dagger}$ Number of additional procedures requiring anesthesia. 
eTable 1. Operational definitions of essential baseline covariates

\begin{tabular}{|c|c|}
\hline Patient characteristics & Operational definitions \\
\hline Age & $\begin{array}{l}\text { Age in years as of the date of the hysterectomy (index date). } \\
\text { Age was categorized as }<40,40-49 \text {, and } \geq 50 \text { years. }\end{array}$ \\
\hline Charlson comorbidity index & $\begin{array}{l}\text { The Charlson Comorbidity Index was to categorize } \\
\text { comorbidities based on the presence of ICD-9 and ICD-10 } \\
\text { diagnosis codes. A weighted sum provided in a single } \\
\text { comorbidity score for a patient. We used a Quan's enhanced } \\
\text { Charlson and Elixhauser comorbidity index. }\end{array}$ \\
\hline Length of stay & $\begin{array}{l}\text { Length of stay refers to the duration of hospitalization and } \\
\text { clinical stay for the index hysterectomy procedure. }\end{array}$ \\
\hline Year & $\begin{array}{l}\text { The calendar year of the index date was divided into early } \\
\text { ( } 2010 \text { to } 2012 \text { ) and late ( } 2013 \text { to } 2015 \text { ) and included in the } \\
\text { model as a dichotomous variable. }\end{array}$ \\
\hline Type of hysterectomy & Identified using ICD9 procedure codes and CPT codes \\
\hline Opioid use disorder & $\begin{array}{l}\text { ICD9 diagnosis codes for opioid use disorder and } \\
\text { buprenorphine and methadone used for medication-assisted } \\
\text { treatment. }\end{array}$ \\
\hline Non-rule-out cancer diagnosis & $\begin{array}{l}\text { Defined as } \geq 2 \text { separate cancer diagnoses occurred } \geq 42 \text { days } \\
\text { apart or } 1 \text { cancer diagnosis with } \geq 1 \text { procedures, including } \\
\text { chemotherapy, radiation, or cancer-related surgery. }{ }^{25}\end{array}$ \\
\hline Chronic opioid use & $\begin{array}{l}\text { To identify chronic opioid use after hysterectomy group-based } \\
\text { trajectory models were used to group together patients with } \\
\text { similar patterns of medication filling during follow-up. The } \\
\text { group with the highest filling probability was classified as } \\
\text { chronic users. }\end{array}$ \\
\hline Psychiatric medications & $\begin{array}{l}\text { Psychotropic medication use during baseline identified using } \\
\text { the American Hospital Formulary System (AHFS) Therapeutic } \\
\text { codes and national drug codes }\end{array}$ \\
\hline $\begin{array}{l}\text { Pain conditions (fibromyalgia, } \\
\text { headache syndromes, back pain) }\end{array}$ & Identified using ICD-9 diagnosis codes \\
\hline $\begin{array}{l}\text { Substance use and abuse (tobacco, } \\
\text { alcohol, marijuana, cocaine, etc.) }\end{array}$ & Identified using ICD-9 diagnosis codes \\
\hline US census region & $\begin{array}{l}\text { US census region refers to the region where patient had the } \\
\text { hysterectomy procedure as defined by the US Census Bureau }\end{array}$ \\
\hline Insurance type & $\begin{array}{l}\text { All patients in the database are insured. There were two types } \\
\text { of insurance: commercial or Medicare. }\end{array}$ \\
\hline \multicolumn{2}{|c|}{ Characteristics of initial opioid prescription } \\
\hline Type of opioid & $\begin{array}{l}\text { The first opioid prescriptions filled were classified as } \\
\text { Oxycodone, Hydrocodone, or other (Codeine, Fentanyl, } \\
\text { Hydromorphone, Meperidine, Morphine, Pentazocine, } \\
\text { Tapentadol, and Tramadol). }\end{array}$ \\
\hline Number of days' supply & $\begin{array}{l}\text { Number of days' supply for the first opioid prescription after } \\
\text { hysterectomy was divided into } 3 \text { categories (1-3 days, 4-7 days, } \\
\text { and } 8 \text { or more days). }\end{array}$ \\
\hline MME tertiles & $\begin{array}{l}\text { The morphine milligram equivalent daily dose for the initial } \\
\text { opioid prescription was determined, and the daily dose was }\end{array}$ \\
\hline
\end{tabular}



thresholds of $\leq 40 \mathrm{mg}, 40.50 \mathrm{mg}$ to $57.86 \mathrm{mg}$ and $\geq 58.33 \mathrm{mg}$ of oral morphine equivalent). 
eTable 2. Comparison of baseline patient characteristics among four trajectory-based model groups, $\mathrm{N}=50,127$

\begin{tabular}{|c|c|c|c|c|}
\hline Patient characteristics & $\begin{array}{c}\text { TBM Group } 1 \\
(\mathrm{~N}=39,677)\end{array}$ & $\begin{array}{c}\text { TBM Group } 2 \\
(\mathrm{~N}=3304)\end{array}$ & $\begin{array}{l}\text { TBM Group } 3 \\
(\mathrm{~N}=6453)\end{array}$ & $\begin{array}{c}\text { TBM Group } 4 \\
(\mathrm{~N}=693)\end{array}$ \\
\hline $\begin{array}{l}\text { LOS in days, mean }(95 \% \\
\text { CI) }\end{array}$ & $\begin{array}{c}2.23(2.20 \\
2.25)\end{array}$ & $\begin{array}{c}2.46(2.34 \\
2.58)\end{array}$ & $\begin{array}{c}2.77(2.68 \\
2.86)\end{array}$ & $\begin{array}{c}3.79(3.39 \\
4.19)\end{array}$ \\
\hline CCI, mean $(95 \% \mathrm{CI})$ & $\begin{array}{l}1.59(1.54 \\
1.63)\end{array}$ & $\begin{array}{c}1.71(1.57, \\
1.85)\end{array}$ & $\begin{array}{c}1.63(1.52 \\
1.75)\end{array}$ & $\begin{array}{l}1.74(1.45 \\
2.02)\end{array}$ \\
\hline Age (years), mean $(95 \% \mathrm{CI})$ & $\begin{array}{c}48.27(48.17 \\
48.38) \\
\end{array}$ & $\begin{array}{c}48.30(47.92 \\
48.67) \\
\end{array}$ & $\begin{array}{c}47.17(46.92, \\
47.42)\end{array}$ & $\begin{array}{c}49.77(48.87, \\
50.67) \\
\end{array}$ \\
\hline $\begin{array}{l}\text { Age at hysterectomy } \\
\text { (years), N (\%) } \\
\quad<40 \\
\quad 40-49 \\
\geq 50\end{array}$ & $\begin{array}{r}7086(17.86) \\
18119(45.67) \\
14472(36.47)\end{array}$ & $\begin{array}{l}666(20.16) \\
1411(42.71) \\
1227(37.14)\end{array}$ & $\begin{array}{l}1348(20.89) \\
3008(46.61) \\
2097(32.50)\end{array}$ & $\begin{array}{l}126(18.18) \\
255(36.80) \\
312(45.02)\end{array}$ \\
\hline $\begin{array}{l}\text { Substance use and abuse, } N \\
(\%) \\
\text { Tobacco use } \\
\text { Alcohol abuse } \\
\text { Other substance abuse }{ }^{\dagger}\end{array}$ & $\begin{array}{c}1907(4.81) \\
117(0.29) \\
41(0.10)\end{array}$ & $\begin{array}{l}230(6.96) \\
12(0.36) \\
3(0.09)\end{array}$ & $\begin{array}{l}500(7.75) \\
48(0.74) \\
11(0.17)\end{array}$ & $\begin{array}{l}60(8.66) \\
9(1.30) \\
4(0.58)\end{array}$ \\
\hline $\begin{array}{c}\text { Psychiatric medications } \\
\text { Benzodiazepines } \\
\text { Antidepressants } \\
\text { Psychostimulants } \\
\end{array}$ & $\begin{array}{c}3071(7.74) \\
7485(18.86) \\
556(1.40) \\
\end{array}$ & $\begin{array}{c}355(10.74) \\
878(26.57) \\
86(2.60) \\
\end{array}$ & $\begin{array}{c}775(12.01) \\
1621(25.12) \\
125(1.94) \\
\end{array}$ & $\begin{array}{c}125(18.04) \\
233(33.62) \\
25(3.61) \\
\end{array}$ \\
\hline $\begin{array}{l}\text { Pain conditions, } \mathrm{N}(\%) \\
\text { Fibromyalgia } \\
\text { Headache syndromes } \\
\text { Back pain }\end{array}$ & $\begin{array}{l}1144(2.88) \\
3012(7.59) \\
3624(9.13)\end{array}$ & $\begin{array}{c}155(4.69) \\
356(10.77) \\
363(10.99)\end{array}$ & $\begin{array}{l}231(3.58) \\
651(10.09) \\
672(10.41)\end{array}$ & $\begin{array}{c}30(4.33) \\
82(11.83) \\
110(15.87)\end{array}$ \\
\hline $\begin{array}{l}\text { Type of hysterectomy, } \mathrm{N} \\
(\%) \\
\text { Vaginal hysterectomy } \\
\text { Laparoscopic/Robotic } \\
\text { Abdominal } \\
\text { hysterectomy }\end{array}$ & $\begin{array}{c}9779(24.65) \\
6113(15.41) \\
23785(59.95)\end{array}$ & $\begin{array}{c}773(23.40) \\
499(15.10) \\
2032(61.50)\end{array}$ & $\begin{array}{c}2205(34.17) \\
816(12.65) \\
3432(53.18)\end{array}$ & $\begin{array}{c}228(32.90) \\
74(10.68) \\
391(56.42)\end{array}$ \\
\hline $\begin{array}{l}\text { US census region, N (\%) } \\
\text { Midwest } \\
\text { Northeast } \\
\text { South } \\
\text { West } \\
\end{array}$ & $\begin{array}{c}11101(27.98) \\
4232(10.67) \\
18700(47.13) \\
5644(14.22) \\
\end{array}$ & $\begin{array}{c}900(27.24) \\
31399.47) \\
1654(50.06) \\
437(13.23) \\
\end{array}$ & $\begin{array}{c}1704(26.41) \\
530(8.21) \\
3288(50.95) \\
931(14.43) \\
\end{array}$ & $\begin{array}{c}186(26.84) \\
66(9.52) \\
332(47.91) \\
109(15.73) \\
\end{array}$ \\
\hline $\begin{array}{c}\text { Insurance type, N (\%) } \\
\text { Commercial } \\
\text { Medicare }\end{array}$ & $\begin{array}{c}36225(91.30) \\
3452(8.70)\end{array}$ & $\begin{array}{l}2964(89.71) \\
340(10.29)\end{array}$ & $\begin{array}{c}5918(91.71) \\
535(8.29)\end{array}$ & $\begin{array}{l}586(84.56) \\
107(15.44)\end{array}$ \\
\hline $\begin{array}{l}\text { Type of health plan, N (\%) } \\
\text { Exclusive provider } \\
\text { organization } \\
\text { Health maintenance } \\
\text { organization } \\
\text { Others } \\
\text { Point of service } \\
\end{array}$ & $\begin{array}{c}4779(12.04) \\
3981(10.03) \\
3646(9.19) \\
27271(68.73)\end{array}$ & $\begin{array}{c}408(12.35) \\
320(9.69) \\
333(10.08) \\
2243(67.89)\end{array}$ & $\begin{array}{c}1,061(12.54) \\
867(10.24) \\
647(7.65) \\
5,888(69.57)\end{array}$ & $\begin{array}{l}70(10.10) \\
89(12.84) \\
85(12.27) \\
449(64.79)\end{array}$ \\
\hline
\end{tabular}




\begin{tabular}{|c|c|c|c|c|}
\hline \multicolumn{5}{|c|}{ Initial Opioid Prescription Characteristics } \\
\hline $\begin{array}{l}\text { Days’ supply (days), mean } \\
(95 \% \mathrm{CI})\end{array}$ & $\begin{array}{l}4.83(4.81 \\
\quad 4.86)\end{array}$ & $\begin{array}{c}4.86(4.76 \\
4.95)\end{array}$ & $\begin{array}{l}4.95(4.88 \\
5.02)\end{array}$ & $\begin{array}{l}5.12(4.89 \\
5.34)\end{array}$ \\
\hline $\begin{array}{l}\text { Avg. MME, mg/day (95\% } \\
\text { CI) }\end{array}$ & $\begin{array}{c}54.37(53.99 \\
54.75)\end{array}$ & $\begin{array}{c}55.93(54.33, \\
57.54)\end{array}$ & $\begin{array}{c}56.04(54.96, \\
57.11)\end{array}$ & $\begin{array}{c}58.39(54.60, \\
62.18)\end{array}$ \\
\hline $\begin{array}{l}\text { Type of Opioid, N (\%) } \\
\text { Hydrocodone } \\
\text { Oxycodone } \\
\text { Other } \\
\end{array}$ & $\begin{array}{c}13697(34.52) \\
22431(56.53) \\
3549(8.94) \\
\end{array}$ & $\begin{array}{c}1241(37.56) \\
1766(53.45) \\
297(8.99) \\
\end{array}$ & $\begin{array}{c}2617(40.55) \\
3237(50.16) \\
599(9.28) \\
\end{array}$ & $\begin{array}{c}266(38.38) \\
354(51.08) \\
73(10.53) \\
\end{array}$ \\
\hline $\begin{array}{l}\text { Days' supply (days), N (\%) } \\
1-3 \\
4-7 \\
\geq 8\end{array}$ & $\begin{array}{c}12703(32.02) \\
23273(58.66) \\
3701(9.33)\end{array}$ & $\begin{array}{c}1048(31.72) \\
1951(59.05) \\
305(9.23)\end{array}$ & $\begin{array}{c}1916(29.69) \\
3874(60.03) \\
663(10.27)\end{array}$ & $\begin{array}{c}193(27.85) \\
423(61.04) \\
77(11.11)\end{array}$ \\
\hline $\begin{array}{l}\text { Tertile MME (mg/day), N } \\
\begin{array}{l}(\%) \\
\quad<40 \\
\quad 40-58.93 \\
\quad \geq 60\end{array}\end{array}$ & $\begin{array}{l}13034(32.85) \\
13412(33.80) \\
13231(33.35)\end{array}$ & $\begin{array}{l}1079(32.66) \\
1102(33.35) \\
1123(33.99)\end{array}$ & $\begin{array}{l}2041(31.63) \\
2204(34.15) \\
2208(34.22)\end{array}$ & $\begin{array}{l}219(31.60) \\
221(31.89) \\
253(36.51)\end{array}$ \\
\hline
\end{tabular}

Abbreviations: TBM=Trajectory based model; $\mathrm{CCl}=$ Charlson Comorbidity Index; LOS=Length of stay; MME=Morphine milligram equivalent;

${ }^{\dagger}$ Other substance use includes marijuana, cocaine, anxiolytic, stimulant, hallucinogenic drugs, or abuse of unspecified drugs/medications.

${ }^{\ddagger}$ Others include Preferred Provider Organization and Indemnity. 


\section{Chronic Opioid Use in Women following Hysterectomy: Patterns and Predictors}

Xuerong Wen, ${ }^{1} \mathrm{PhD}, \mathrm{MPH}$, Stephen Kogut, ${ }^{1} \mathrm{PhD}$, Hilary Aroke, ${ }^{1} \mathrm{MD}, \mathrm{PhD}, \mathrm{MPH}, \mathrm{Lynn}$ Taylor ${ }^{1}$, $\mathrm{MD}$, Kristen A. Matteson, ${ }^{2} \mathrm{MD}, \mathrm{MPH}$,

1. Health Outcomes Research, Department of Pharmacy Practice, College of Pharmacy, University of Rhode Island.

2. Obstetrics and Gynecology, Women \& Infants Hospital and the Warren Alpert Medical School, Brown University. 


\begin{abstract}
Background Most women are prescribed an opioid at hospital discharge-after hysterectomy. The objective of this study was to determine the association between initial opioid prescribing characteristics and chronic opioid use after hysterectomy.
\end{abstract}

Methods: This study included women enrolled in a commercial health plan who had a hysterectomy between July 01, 2010 and March 31, 2015. We used trajectory models to define chronic opioid use as patients with the highest probability of having an opioid prescription filled during the 6 months post-surgery. We used multivariable logistic regression to examine the association between initial opioid dispensing (amount prescribed and duration of treatment) and chronic opioid use after adjusting for potential confounders.

Results: A total of $434 \underline{693}$ of $49,84450,127$ ( $0.91 .38 \%)$ opioid naïve women met the criteria for chronic opioid use following hysterectomy. The baseline variables and initial opioid prescription characteristics predicted the pattern of long-term opioid use with moderate discrimination (c statistic $=0.73 \underline{0.70})$. Significant predictors of chronic opioid use included initial opioid daily dose ( $\geq 60$ MME vs < $40 \mathrm{MME}$, aOR: $1.70 \underline{1.43}, 95 \% \mathrm{Cl}: 1.28 \underline{1.14-2.261 .79}$ ), and days' supply 4-7 days vs $1-3$ days, aOR: $1.41 \underline{1.28}, 95 \% \mathrm{Cl}: 1.11 \underline{1.06}-1.791 .54 ; \geq 8$ days vs $1-3$ days, aOR: 2.041 .41 , 95\% $\%$ : 1.431.05-2.901.89). Other significant baseline predictors included older age, abdominal or laparoscopic/robotic hysterectomyhysterectomy type, tobacco use, co-morbiditiespsychiatric medication use, back pain, and fibromyalgiaheadache.

Conclusion: Initial opioid prescribing characteristics are associated with risk of chronic opioid use after hysterectomy. Prescribing lower daily doses and shorter days' supply of opioids to women after hysterectomy may result in lower risk of chronic opioid use. 


\section{Introduction}

The rapid increase in the incidence of opioid-related overdoses and deaths has become a major public health issue-crisis in the United States. Overdose and death from prescription opioid overdose increased $400 \%$ in women between 1999 and $2013 .{ }^{1-3}$ For many women, their first exposure to prescription opioids often occurs during the post-operative period, which makes this a potential target for strategies to reduce the risk of chronic opioid use..$^{4-6}$ Several observational studies suggest that surgery is a risk factor for chronic opioid use. ${ }^{5-18}$ Two studies have examined the relationship between the initial opioid prescribing characteristics and chronic opioid use in the postoperative setting and arrived at contradictory conclusions. ${ }^{6,12}$ One study reported that initial exposure to prescription opioids after minor surgery increases the risk of chronic opioid use by $44 \%,{ }^{12}$ while the other suggested that the initial opioid prescribing characteristics, including type of opioid dispensed, days' supply, or daily dose (in morphine milligram equivalents), were not associated with chronic opioid use after cesarean delivery. ${ }^{6}$

Hysterectomy, the most commonly performed non-obstetric surgery among women in the United States, ${ }^{19-21}$ poses a potential risk for chronic opioid use because an estimated $82 \%$ of hysterectomy patients receive an opioid prescription at hospital dischargeafter the hysterectomy surgery. ${ }^{20,22}$ However, the relationship between the initial opioid prescribing characteristics and chronic opioid use after hysterectomy remains largely unknown. The objective of this study was to identify baseline risk factors for chronic opioid use after hysterectomy and examine the association between initial opioid prescribing characteristics after hysterectomy and subsequent chronic use of opioids among opioid naïve women.

\section{Methods}

Data Sources: Study data were derived from the national Optumlnsight Clinformatics Data Mart $^{\mathrm{TM}}$ (Optumlnsight, Eden Prairie, MN). The Optum Clinformatics Data Mart is an 
administrative health claims database from a large national insurer which includes approximately 35 million beneficiaries. The dataset contains transactional reimbursement data for health care utilization including outpatient pharmacy dispensing, and inpatient and outpatient medical claims. ${ }^{23}$ The administrative enrollment file has eligibility information, the outpatient pharmacy file has the national drug code (NDC) for each drug dispensed, and the medical files have the Current Procedural Terminology (CPT) code for medical procedure, and International Classification of Diseases, Ninth Revision, Clinical Modification (ICD-9-CM) code for medical procedure and diagnosis. Patients included in the database had both medical and prescription coverage. The data was used under license agreement between the University of Rhode Island and Optumlnsight Inc.

Study Population: This study included women ( $\geq 18$ years) who had a hysterectomy between July 01, 2010 and March 30, 2015. Women were required to have at least 6 months of continuous enrollment prior to hysterectomy. To identify an opioid-naïve study cohort, we excluded women with a diagnosis of opioid use disorders or any opioids filled 6 months before the date of the hysterectomy (index date) (eTable 1). The hysterectomy procedures were identified using ICD-9-CM procedure and CPT codes from both the inpatient and outpatient claims (eTable 1). ${ }^{21,24}$ We excluded patients who had a radical hysterectomy and patients for whom we could not rule-out a cancer diagnosis which was defined as $\geq 2$ separate medical claims with a cancer diagnosis occurred $\geq 42$ days apart or receipt of chemotherapy, radiation, or other cancer-related surgery (eTable 1). ${ }^{25}$ Women who filled at least one opioid prescription at a retail pharmacy within 7 days of discharge were considered exposed to prescription opioids and included in the final study cohort. The 7-day window was based on the assumption that an opioid prescription filled by an opioid naïve individual during this period was likely used to treat acute pain after hysterectomy. This study was approved by the University of Rhode Island Institutional Review Board (IRB\#957873-2). 
Exposure to opioids: Opioid prescriptions were identified using national drug codes (NDC) from the outpatient pharmacy claims. Opioid prescriptions were classified as hydrocodone, oxycodone and others (including codeine, fentanyl, hydromorphone, meperidine, morphine, pentazocine, tapentadol, and tramadol). Duration of the initial prescription was categorized as 1$3,4-7, \geq 8$ days. We calculated the morphine milligram equivalent (MME) for the initial opioid prescription using the Center for Disease Control (CDC) conversion Tables (2016 version). The average daily MME dose was categorized into tertiles ( $\leq 40 \mathrm{mg} / \mathrm{day}, 40.50-58.9 \mathrm{mg} / \mathrm{day}$, and $\geq 60 \mathrm{mg} /$ day).

Outcome Assessment: We used trajectory models to generate our dichotomous primary study outcome as (chronic opioid use after hysterectomy present or absent ${ }^{11}$ The trajectory models allow the use of observed longitudinal data to determine distinct opioid prescription filling patterns in the study population during the six months period after hysterectomy. This approach classifies patients into groups with similar opioid prescription filling patterns during follow-up without relying on a priori and often subjective-prespecified cutoff value for the definition of chronic opioid use. ${ }^{26,27}$ In order to classify the trajectory groups for opioid use during the defined follow-up window (6 months after hysterectomy), we first generated 6 dichotomous variables to indicate if a study participant filled a prescription of an opioid analgesic during each of 6 consecutive 30 -day follow-up periods, e.g., month 1 is from 7 to 37 days, month 2 is from 37 to 67 days, and month 6 is from 157 to 187 days. ${ }^{11}$ We then modeled these 6 binary indicators of opioid use in each 30-day follow-up period as a longitudinal response in a logistic group-based trajectory. ${ }^{28,29}$ Using the trajectory model, we estimated the probability of membership of patients in each group, and the probability of filling a opioid prescription over time as a smooth function of time. The model was fitted using 2 to $6 \underline{5}$ opioid exposure groups and the number of groups was chosen based on the value of the Bayesian Information Criterion. ${ }^{30}$ In each group, a thirdorder polynomial (including linear, squared, and cubic terms) of time were used to model the probability of filling a prescription for opioids. Patients were assigned to the trajectory group in 
which they had the highest probability of membership. Based on the model results, the trajectory group with the highest probability of filling any opioid prescription beyond the initial prescription was defined as "chronic opioid users". All other trajectory groupings were classified as non-chronic opioid users. The percentage of patients who filled an opioid prescription was reported in each trajectory for every consecutive 30-day follow-up period.

Potential Predictors of Chronic Opioid Use: We collected data on potential confounding variables that may be related to both initial opioid prescribing characteristics and chronic opioid use after hysterectomy. ${ }^{6-11,31}$ Previous studies demonstrated that age, certain pain conditions, and psychiatric disorders are associated with both postsurgical chronic pain and chronic prescription opioid use..$^{6,21,31-36}$ Covariates assessed in our analyses included age at hysterectomy, Charlson comorbidity index, hospital length of stay, smoking status, use of alcohol and illicit substances, psychiatric medication use, pre-operative pain conditions, types of hysterectomy procedure performed, US census region, type of insurance (Medicare or private insurance) and health plan (exclusive provider organization, health maintenance organization, point of service, and others including indemnity and preferred provider organization), year of surgery, and initial opioid prescribing characteristics (medication type, average daily dose, and number of days' supplied).

Statistical Analysis: Categorical variables were examined and compared using Chi-squared or Fisher exact tests. Continuous variables were compared using the student $t$ test. Statistical tests and modeling were conducted to identify risk factors for chronic opioid use among women after hysterectomy. A multivariable logistic regression model was used to estimate the association between baseline demographic and clinical variables, initial opioid prescribing characteristics, and chronic opioid use after hysterectomy among opioid-naive patients who filled at least one opioid prescription within 7 days after hysterectomy.

\section{Sensitivity studies were conducted using multivariate logistic regression models to} examine if the significant association between initial prescribing pattern and subsequent chronic 
opioid use were upheld with a different definition of chronic opioid use post hysterectomy or a more restricted definition for opioid naivety after adjusting for other covariates.

A probability of type 1 error (alpha) $=0.05$ was set as the threshold of statistical significance. The trajectory models were conducted using "Proc Traj". All statistical analyses were performed using Statistical Analysis Software (SAS), version 9.4 (SAS Institute Inc, Cary, NC).

\section{Results}

Study cohort: A total of 157,934 women underwent hysterectomy between 07/01/2010 and 03/31/2015. Of these patients, $22,186(14 \%)$ patients did not meet the continuous enrollment criteria, $14,696(9 \%)$ had suspected cancer diagnoses or radical hysterectomy, $23,70718,202$ $(12 \%)$ didn't fill an opioid prescription within 7 days of surgery, $3,8103,918 \underline{(2 \%)}$ were missing important demographic data, 43,30348,778 (31\%) used opioids in the 6 months prior to surgery and $232(0.1 \%)$ were diagnosed with OUD within 6 months prior to hysterectomy leaving $49,84450,127(32 \%)$ patients for the analysis. In the final study cohort, only 3 patients lost eligibility and were censored during the 6-month follow-up period. A total of 12,966-985 (26\%) patients had an abdominal hysterectomy; 29,409640 (59\%) patients had a laparoscopic or robotic assisted laparoscopic hysterectomy; and 7,469-502 (15\%) patients had a vaginal hysterectomy (Figure 1).

The trajectory model identified 4 distinct trajectories of prescription opioid use after hysterectomy, with total 39,677 (79.15\%) patients in trajectory $1,3,304(6.59 \%)$ in trajectory 2 , $\underline{6,453}(12.87 \%)$ in trajectory 3, and $693(1.38 \%)$ in trajectory 4. (Figure 2). Among the patients in trajectory $4(\mathrm{n}=434 \underline{693}, 0.87 \underline{1.38} \%), 70 \underline{57} \%, 60 \underline{55} \%, 68 \underline{57} \%, 76 \underline{55} \%, 72 \underline{48} \%$, and $53 \underline{41} \%$ filled an opioid prescription at 1 month, 2 months, 3 months, 4 months, 5 months, and 6 months following an initial prescription in the 7 days post-hysterectomy, respectively. Patients in trajectory 4 were classified as chronic opioid users. No patient in trajectory 1 ( $n=39,677$, 
79.15\%) filled any opioid prescription (0\%) from month 1 to 6 . Among patients in trajectory $1-2$ $(\mathrm{n}=2,808 \underline{3,304}, 5.6 \underline{69} \%), 500.3 \%$ filled a prescription at month 1 , which increased to $2.6 \%$ at month $2,8 \%$ at month 3 , and then followed by $10 \%$ with opioid fills for the subsequent 3 monthsgradually decreased in each subsequent month to $0 \%$ with an opioid prescription fill at month 5. Thirty-twa-five percent of patients in trajectory $2 \underline{3}(n=2,543 \underline{6,543}, 5.1 \underline{12.87} \%)$ filled an opioid prescription at month 1 , which decreased to $5 \underline{8} \%$ at month 2 , and then gradually increased-decreased to from $4 \%$ at month 3 , and to $29 \underline{3} \%$ at months $\underline{4,5}$, and 6 . Among patients seen in trajectory $3(n=44,059,88.4 \%), 24 \%$ filled an opioid prescription at month 1 followed by $0 \%$ with opioid fills for the subsequent 5 months. A description of the demographic and clinical characteristics of the patients assigned to each of these 4 trajectories is included in eTable2.

Characteristics of patients and initial prescriptions: Among the total 49,84450,127 opioid naïve patients who filled at least one opioid prescription within 7 days after hysterectomy, 434 $\underline{693}(0.871 .38 \%)$ patients were categorized as chronic opioid users and 49,41048,434(99.1398.62\%) were categorized as non-chronic users. Baseline characteristics among chronic opioid users after hysterectomy were significantly different from non-chronic opioid users (Table 1). Compared to the women without chronic opioid use, women with chronic opioid use were older and more likely to have a higher Charlson comorbidity index, a history of tobacco use, and a history of alcohol use. Chronic opioid users also were also more likely to be taking psychiatric medications, including benzodiazepines, antidepressants, and stimulants. Back pain and headache syndromes fibromyalgia-were more prevalent in the chronic opioid use group. Compared to women with commercial insurance, women with Medicare insurance had higher rates of chronic opioid use. About 1.5 percent of Medicare patients who were chronic opioid users after hysterectomy were younger than 65 .

the average daily MME/day and the type of the initial opioid prescription were similar between women categorized as having chronic use and not having 
chronic opioid use. The days' supplied of the initial opioid was marginally greater among chronic users than for non-chronic users (5.12 versus 4.85 days, respectively, $p=0.0205$ ) (Table 1$)$. Overall, oxycodone was more frequently prescribed hydrocodone or other opioids. Predictors of chronic opioid use among all patients in the study: The results of multivariable logistic regression analyses are shown in Table 2. A number of factors were significantly associated with chronic opioid use after hysterectomy including $\_$lder age, geographic region, abdominal or laparoscopic/robotic hysterectomymode of hysterectomy, Charlson comorbidity index, tobacco use, alcohol use, and prescribed psychotropic medicationsother substance use, benzodiazepines use, antidepressants use, antipsychotics Pain conditions, including headache syndromes fibromyalgia-and back pain at baseline, were also identified as significant risk factors for chronic opioid use after hysterectomy. Health plan type, census region, surgical setting, fibromyalgia, and other substance use disorders were not included in the final multivariable analyses due to lack of significance.

The characteristics of the initial opioid prescription, including type of opioid dispensed, days' supply, and daily dose in MME, were fitted in the model. Days' supply and daily dose in MME were divided into their tertiles. Among initial opioid prescribing characteristics, significant predictors of chronic opioid use were initial prescription of hydrocodone (compared to oxycodone, aOR: $1.33 \underline{31}, 95 \% \mathrm{Cl}: 1.11 \underline{10}-1.59 \underline{57})$, days' supply (4-7 days vs $\leq 3$ days, aOR: 1.3528, 95\%Cl: 1.11므-1.7954; $\geq 8$ days vs $\leq 3$ days, aOR: $2.041 .41,95 \% \mathrm{Cl}: 1.43 \underline{05}-2.901 .89$ ), and daily dose in MME ( $\geq 60 \mathrm{mg} /$ day vs $\leq 40 \mathrm{mg} /$ day, aOR: $1.70 \underline{43}, 95 \% \mathrm{Cl}: 1.28 \underline{14}-2.26 \underline{1.79}$ ), The C-statistic for the fitted full model was $0.73 \underline{70}$, indicating moderate predictability (Table 2 ) The frequencies of post-surgery complications and other conditions associated with pain during the 6-month follow-up were examined in the study cohort (Table 3). Compared with nonchronic opioid users, women who developed chronic opioid use after hysterectomy were more 


\begin{abstract}
frequently diagnosed with chronic pancreatitis, headache syndrome, back pain, fibromyalgia, and underwent additional anesthetic procedures.
\end{abstract}

\title{
Sensitivity Studies
}

We redefined chronic opioid use as a total duration of opioid use (total days' supply) that exceeded 90 days during 6-month follow-up period, finding that the days supplied and MME of the initial opioid prescription were associated with chronic opioid use according to this definition. An initial days supply was associated with an adjusted 1.81 fold increase in chronic use as compared with a supply of less than 3 days $(95 \% \mathrm{Cl}: 1.21-2.70)$; while an initial MME/day of 60 or greater was associated with an adjusted 1.37 fold increase in chronic use (95\% Cl: $1.00-1.87)$ as compared with an initial MME of $\leq 39.64 \mathrm{mg} / \mathrm{day})$. These results are similar as the results which defined chronic use using trajectory models.

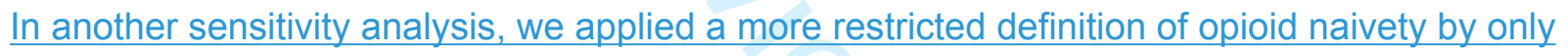
including patients having 12-month continuous eligibility and without any opioid dispensing or diagnosis of OUD during the 12 months prior to hysterectomy. In this analysis, the highest tertile of MME/day (exceeding $58.92 \mathrm{mg}$ ) for the initial opioid prescription remained predictive of subsequent chronic opioid use as compared with the lowest tertile of $\leq 39.64 \mathrm{mg} / \mathrm{day}$ (aOR: 1.38; $95 \% \mathrm{Cl}: 1.07-1.80) ;$ while the trend for days supplied also remained consistent, albeit failing to reach statistical significance. (aOR: $1.38,95 \% \mathrm{Cl}: 1.0-1.90$ for $8+$ days' supply vs $\leq 3$ days' supply)

\section{Discussion}

Hysterectomy is the most common surgical procedure among non-pregnant women. In our study, among the $49,844 \underline{50,127}$ opioid naïve women who had a hysterectomy and were dispensed at least one opioid prescription within 7 days after hysterectomy, $0.87 \underline{1.38} \%$ became chronic opioid users during the within 6 months after surgery. Several demographic factors, 
such as age, mode of hysterectomy, tobacco use, alcohol use, and psychiatric use were associated with chronic opioid use after hysterectomy. More importantly, however, we found that characteristics of the initial opioid prescription, including type, dose, and duration affected the probability of chronic opioid use An initial supply of 8 or more days, and an initial MME of at least $60 \mathrm{mg}$ per day were each associated with a greater than $40 \%$ increase in the risk of Subsequent chronic use of opioids (Table 2). Each year in the United States, there are approximately 430,000 inpatient hysterectomy cases. ${ }^{37}$ The findings of this study suggest that lessening the intensityduration and amount of opioids in the initial focusing on these easily modifiable opioid prescription haracteristics may lead to decrease in the risk incidence of chronic opioid use after hese easily modifiable opioid prescription characteristics to decrease the incidence of chronic opioid use after this prevalent surgical procedure.

Multiple demographic and clinical factors were associated with increased risk of chronic opioid use after hysterectomy, including older age, abdominal or laparoscopic/robotic hysterectomymode of hysterectomy, comorbidities, tobacco use, substance abuseuse $\underline{\text { disorders, }}$, certain pain conditions, and use of prescription any psychiatric medications use (Table 2). Our findings were similar to other studies that showed that these factors increase the risk of chronic opioid use after major cardiac, thoracic, abdominal and pelvic procedures, or specific surgeries, including cesarean delivery, hysterectomy, hip or knee arthroplasty, spine, or bariatric surgeries. ${ }^{5-17,} 38,39$ Previous studies have reported that residing in the South or Midwest regions of the U.S. was associated with prescription opioid abuse specifically among women. In our study, the risk of chronic opioid use after hysterectomy was also higher in the South, Midwest, and West compared with the Northeast.(Table 2) These findings underscore the importance of considering the individual patient's pain management needs ${ }_{2}$-and-risk factors for opioid misuse at the time of opioid prescribing after hysterectomy and other surgical procedures, and importance of medical care, monitoring and follow-up post-operatively. 
The literature describing the relationship between the initial opioid prescribing characteristics and the risk of chronic opioid use after hysterectomy among opioid naïve patients is limited. Prior observational studies have produced inconclusive results, which may be attributed to the study sample (inclusion of patients with minor surgical procedures) or small study sample size. ${ }^{11,38}$ Our results showed that initial opioid prescribing characteristics, including opioid type, daily dose, and duration of days' supply were associated with chronic opioid use in the 6 months following hysterectomy among opioid naïve patients. The odds of chronic opioid use following initiation after hysterectomy were $3531 \%$ higher among patients starting hydrocodone compared to patients who began therapy with oxycodone even though prior research has demonstrated that oxycodone is associated with risk of addiction, morbidity and mortality. ${ }^{40-42}$ One explanation for this finding is that for many years hydrocodone was the most commonly prescribed opioid medication in the United States, in part because it was a schedule III drug during the study period and was considered to be lesser risk than schedule II opioids such as oxycodone, therefore, hydrocodone may have been prescribed more liberally, especially to patients with a higher tendency of opioid overuse, which is a possible confounding by contraindication. In August 21, 2014, the U.S. Drug Enforcement Administration (DEA) issued stricter prescribing requirements and moved hydrocodone-containing medications from a Schedule III to a Schedule II controlled substance..$^{43}$ Notably, the initial opioid prescribing characteristics, such as longer days' supply and higher daily morphine milligram equivalent dose, that were associated with post-operative chronic opioid use after hysterectomy, are also risk factors for opioid misuse and opioid-related mortality. ${ }^{44-47}$ By identifying modifiable risk factors for chronic opioid use after hysterectomy, such as pre-operative opioid prescribing, abdominal or laparoscopic/robotic hysterectomymode of hysterectomy, and characteristics of opioid prescribing after surgery, we may be able to develop strategies and interventions to decrease the likelihood of chronic opioid use. 
There is no standard definition for chronic opioid use after surgery. We selected the trajectory modeling approach because of its advantages over other methods for describing longitudinal trajectories and identifying patients who used opioids consistently over a longer duration. ${ }^{29,30}$ Traditional medication adherence measures, such as the proportion of days covered or the number of months of continuous medication use, may not distinguish between consistent users in follow-up periods or between patients who discontinue medication completely versus those who simply have a gap in use. The trajectory models repeatedly assess medication use throughout the entire follow-up period and summarize long-term medication adherence accounting for the time-varying nature of adherence. ${ }^{29}$ We found that $0.91 .38 \%$ of hysterectomy patients became chronic opioid users in the 6 months after surgery. Using Optum data from January 01, 2011 to December 31, 2014, Swenson et al. reported 0.5\% (122 out of 24,331$)$ of women who had a hysterectomy had new persistent opioid use following hysterectomy. ${ }^{5}$ In Swenson's study, persistent opioid use in the 6 months post-hysterectomy was defined as a minimum of 2 opioid prescriptions in the 6 months post-surgery (one between 15-90 days and one at least $91-180$ days) and top quartile use (amount and duration). ${ }^{5}$ The difference in incidence of chronic use/new persistent use between these studies is attributable to the use of different definitions for chronic opioid use.

Our findings suggest that prescribing lower doses for shorter days' supply may risk of subsequent chronic opioid use,-be a better strategy for post-surgical pain management, andwhich is consistent with the strategies published in guidelines advocated by multiple federal and state agencies, professional societies, and advocacy groups. Although specific guidelines differ in exact wording and specific recommendations, prescribing the lowest dose for the shortest amount of time and screening for substance use and opioid dependence are clearly defined themes across multiple guidelines. ${ }^{48-50}$ However, practice change solely on the basis of guidelines can be slow, and current evidence suggests that physicians are prescribing excess opioids to patients to control acute pain post-surgery at hospital discharge. Studies from the 
surgical fields of urology, orthopedics, and obstetrics and gynecology have shown that physicians prescribe more than twice the amount of opioid medication patients actually consume post-discharge which adds up to millions of excess unused opioid tablets available for diversion and abuse. ${ }^{51-54} \mathrm{~A}$ recent study showed that among obstetricians and gynecologists, only $62 \%$ reported tailoring prescriptions to the individual patient and only $22 \%$ reported they performed an opioid dependence screen prior to prescribing. ${ }^{55}$ Improving adherence to best practices for opioid prescribing, including tailoring prescriptions to the individual patient's pain management needs and risks of future opioid misuse and abuse, could play a major role in reducing the magnitude of the opioid epidemic.

\section{Limitations}

First, the study subjects included in this study are commercially insured in the United States with $109 \%$ covered by Medicare Advantage (Table1). The demographic characteristics, clinical conditions, and opioid preoperative use patterns may differ from uninsured, Medicaid or Medicare covered populations. Thus, the study results might not be generalizable to patients enrolled in Medicaid or Medicare. with government covered-jinsurances.

Our study is subject to several limitations due to the nature of observational studies using claims data. Some important medical indications, such as severity of pain, which may be related to the hysterectomy procedure or underlying conditions leading to hysterectomy, were not captured in the data. Other unmeasured confounding factors included social and economic factors during follow up. The low prevalence of OUDs or methadone use excluded in figure 1. and other substance abuseuse disorders, tobacco use, and alcohol abuse presented in table 1 might be due to poor sensitivity of ICD-9 diagnosis codes or CPT codes._However, the impact may be non-differential if the unmeasured variables are similarly distributed in two comparison groups. 
Opioid analgesics were assessed using pharmacy claims, which only captured opioid medications legally obtained and filled at outpatient pharmacies. The $69 \%$ prevalence of Patients not filling the initial opioid prescription post-hysterectomy may be attributed to either some patients-not using opioids for post-surgical pain or to patients-receiving their opioid prescription from an inpatient pharmacy. The actual patterns of opioid use (actual consumption) was not measured and, based on prior studies, differs substantially from the amount of opioid medication prescribed. Furthermore, the result that hydrocodone has a higher risk of chronic use after hysterectomy compared to oxycodone could be due to a possible confounding by contraindication.

The outcome assessed in this study was chronic opioid use during the first 6 months after hysterectomy. Although chronic opioid use within 6 months has been associated with opioid misuse and opioid-related death, it was not possible to differentiate whether patients persistently used opioids for 6 months as a legitimate treatment for pain control or because they became dependent upon opioids after their medications to treat acute pain. ${ }^{56}$ Additionally, it was not possible is impossible to know if the women were continuing to receive their opioid for the pain related to their hysterectomy or for other pain; the reason for the opioid prescription fills during this time periods; - It is possible that the reason for filling opioid prescriptions in the 6 months following hysterectomy was not for personal use but for diversion. 57 Our findings that chronic opioid use post hysterectomy was significantly associated with preoperative opioid use and initial opioid prescribing could be due to complex relationships between chronic pain post-surgery, acute pain post-surgery, and chronic pain pre-surgery.

\section{Conclusions}




\section{In this study, About $0.87 \%$ ofapproximately 3 in 200 opioid naïve women appear to} become chronic opioid users after hysterectomy. Besides commonly recognized demographic and clinical risk factors, chronic opioid use after hysterectomy was associated with initial opioid prescribing characteristics, such as longer days' supply and higher daily opioid dose. Although prescription opioid medications provide effective analgesia after surgery, they must be used with caution given the potential risk for subsequent chronic opioid use that is associated with opioid misuse and overdose-related mortality. Our findings support the need to manage post-surgical pain with the least amount of opioid medication possible to effectively control a patient's symptoms, and close clinical post-op follow-up for those patients who prescribed opioids. 


\section{References}

1. The U.S. Department of Health and Human Services Office on Women's Health. White Paper: Opioid Use, Misuse, and Overdose in Women.

https://www.womenshealth.gov/files/documents/white-paper-opioid-508.pdf. Accessed 01/18/17.

2. McCarthy M. Opioid overdose deaths rose fivefold among US women in 10 years. BMJ 2013;347:f4415.

3. Chou R, Ballantyne JC, Fanciullo GJ, Fine PG, Miaskowski C. Research Gaps on Use of Opioids for Chronic Noncancer Pain: Findings From a Review of the Evidence for an American Pain Society and American Academy of Pain Medicine Clinical Practice Guideline. The Journal of Pain, Vol 10, No 2 (February), 2009: pp 147-159

4. An analysis of the impact of opioid overprescribing in America. United States for nondependence. The role of opioids in treating postsurgical pain. Pg 9-13. https://www.planagainstpain.com/wpcontent/uploads/2017/09/PlanAgainstPain USND.pdf. The Plan Against Pain. September 26, 2017. Accessed December 20, 2018.

5. Swenson CW, Kamdar NS, Seiler K, Morgan DM, Lin P, As-Sanie S. Definition development and prevalence of new persistent opioid use following hysterectomy. Am J Obstet Gynecol. 2018 Nov;219(5):486.e1-486.e7.

6. Bateman BT, Franklin JM, Bykov K, et al. Persistent opioid use following cesarean delivery: patterns and predictors among opioid-naïve women. Am J Obstet Gynecol. 2016; S0002-9378(16)00478-6.

7. Hansen CA, Inacio MC, Pratt NL, Roughead EE, Graves SE. Chronic Use of Opioids Before and After Total Knee Arthroplasty: A Retrospective Cohort Study. J Arthroplasty. 2016 Oct 4. 
8. Clarke H, Soneji N, Ko DT, Yun L, Wijeysundera DN. Rates and risk factors for prolonged opioid use after major surgery: population based cohort study. BMJ. 2014;348:g1251.

9. Hetmann F, Kongsgaard UE, Sandvik L, Schou-Bredal I. Prevalence and predictors of persistent post-surgical pain 12 months after thoracotomy. Acta Anaesthesiol Scand. 2015;59(6):740-748.

10. Sun EC, Darnall BD, Baker LC, Mackey S. Incidence of and Risk Factors for chronic opioid use among opioid-naive patients in the postoperative period. JAMA Intern Med. 2016 Sep 1;176(9):1286-93.

11. Raebel MA, Newcomer SR, Reifler LM, Boudreau D, Elliott TE, DeBar L, Ahmed A, Pawloski PA, Fisher D, Donahoo WT, Bayliss EA. Chronic use of opioid medications before and after bariatric surgery. JAMA. 2013 Oct 2;310(13):1369-76.

12. Alam A, Gomes T, Zheng H, Mamdani MM, Juurlink DN, Bell CM. Long-term analgesic use after low-risk surgery: a retrospective cohort study. Arch Intern Med. 2012;172(5):425-430.

13. Calcaterra SL, Scarbro S, Hull ML, Forber AD, Binswanger IA, Colborn KL. Prediction of Future Chronic Opioid Use Among Hospitalized Patients. J Gen Intern Med. 2018 Jun;33(6):898-905. doi: 10.1007/s11606-018-4335-8. Epub 2018 Feb 5.

14. Rao AG, Chan PH, Prentice HA, Paxton EW, Navarro RA, Dillon MT, Singh A. Risk factors for postoperative opioid use after elective shoulder arthroplasty. J Shoulder Elbow Surg. 2018 Jun 8.

15. Jafari A, Shen SA, Bracken DJ, Pang J, DeConde AS. Incidence and predictive factors for additional opioid prescription after endoscopic sinus surgery. Int Forum Allergy Rhinol. 2018 May 31. 
16. Namba RS, Singh A, Paxton EW, Inacio MCS. Patient Factors Associated With Prolonged Postoperative Opioid Use After Total Knee Arthroplasty. J Arthroplasty. 2018 Apr 9.

17. Pugely AJ, Bedard NA, Kalakoti P, Hendrickson NR, Shillingford JN, Laratta JL, Saifi C, Lehman RA, Riew KD. Opioid use following cervical spine surgery: trends and factors associated with long-term use. Spine J. 2018 Apr 10. pii: S1529-9430(18)30129-3.

18. Dunn LK, Yerra S, Fang S, Hanak MF, Leibowitz MK, Tsang S, Durieux ME, Nemergut EC, Naik BI. Incidence and Risk Factors for Chronic Postoperative Opioid Use After Major Spine Surgery: A Cross-Sectional Study With Longitudinal Outcome. Anesth Analg. $2018 \mathrm{Jul} ; 127(1): 247-254$.

19. Merrill RM. Hysterectomy surveillance in the United States, 1997 through 2005. Med Sci Monit. Jan; 2008 14(1):CR24-31.

20. Darnall B, Li H. Hysterectomy and predictors for opioid prescription in a chronic pain clinic sample. Pain Med. 2011 Feb;12(2):196-203.

21. Merrill RM, Layman AB, Oderda G, Asche C. Risk estimates of hysterectomy and selected conditions commonly treated with hysterectomy. Ann Epidemiol. Mar; 2008 18(3):253-260.

22. Brandsborg B, Nikolajsen L, Hansen CT, Kehlet H, Jensen TS. Risk factors for chronic pain after hysterectomy: a nationwide questionnaire and database study. Anesthesiology. May; 2007 106(5): 1003-1012.

23. Optum. Retrospective database analysis. Optum Inc. 2013. Available at: https://www.optum.com/content/dam/optum/resources/productSheets/RetrospectiveDatabase-Analysis.pdf. Accessed 01/28/2017.

24. Keshavarz H, Hillis SD, Kieke BA. Hysterectomy surveillance-United States, 19941999. MMWR. 2002;1(SS-5):1-8. 
25. Chastek B, Harley C, Kallich J, Newcomer L, Paoli CJ, Teitelbaum AH. Health care costs for patients with cancer at the end of life. J Oncol Pract. 2012 Nov;8(6):75s-80s.

26. Jones BL, Nagin DS. Advances in group-based trajectory modeling and an SAS procedure for estimating them. Socio Meth Res 2007; 35(4): 542-571.

27. Schwarz G. Estimating the dimension of a model. Ann Stat 1978; 6(2): 461-464.

28. Nagin DS. Analyzing developmental trajectories: a semiparametric, group-based approach. Psychol Methods 1999;4:139.

29. Franklin JM, Shrank WH, Pakes J, et al. Group-based trajectory models: a new approach to classifying and predicting long-term medication adherence. Med Care 2013;51:789-96.

30. Jones BL, Nagin DS. Advances in group-based trajectory modeling and an SAS procedure for estimating them. Socio Meth Res 2007; 35(4): 542-571.

31. Turk DC, Okifuji A. What factors affect physicians' decisions to prescribe opioids for chronic noncancer pain patients? Clin J Pain. Dec; 1997 13(4):330-336.

32. Sullivan MD, Edlund MJ, Zhang L, Unutzer J, Wells KB. Association between mental health disorders, problem drug use, and regular prescription opioid use. Arch Intern Med. Oct 23; 2006 166(19):2087-2093.

33. Hooten WM, Townsend CO, Bruce BK, et al. Effects of smoking status on immediate treatment outcomes of multidisciplinary pain rehabilitation. Pain Med. Mar; 2009 $10(2): 347-355$.

34. Rudd RA, Aleshire N, Zibbell JE, Gladden MR, Increases in Drug and Opioid Overdose Deaths - United States, 2000-2014.

http://www.cdc.gov/mmwr/preview/mmwrhtml/mm6450a3.htm. Accessed 01/26/18.

35. Brandborg B, Dueholm M, Nikolajsen L, Kehlet H, Jensen TS. A prospective study of risk factors for pain persisting 4 months after hysterectomy. Clin J Pain. 2009 May;25(4):263-8. 
36. Calderon M, Castorena G, Pasic E. Postoperative pain management after hysterectomy - a simple approach. http://cdn.intechopen.com/pdfs/35360/InTech-

\section{Postoperative pain management after hysterectomy a simple approach.pdf.}

\section{Accessed 01/24/17.}

37. Wright JD, Herzog TJ, Tsui J, et al. Nationwide trends in the performance of inpatient hysterectomy in the United States. Obstet Gynecol. 2013;122(2 Pt 1):233-41.

38. Kim SC, Choudhry N, Franklin JM, Bykov K, Eikermann M, Lii J, Fischer MA, Bateman BT. Patterns and predictors of persistent opioid use following hip or knee arthroplasty. Osteoarthritis Cartilage. 2017 Sep;25(9):1399-1406. doi: 10.1016/j.joca.2017.04.002. Epub 2017 Apr 19.

39. Soneji N, Clarke HA, Ko DT, Wijeysundera DN. Risks of developing persistent opioid use after major surgery. JAMA Surg. 2016 Nov 1;151(11):1083-1084. doi:

\subsection{1/jamasurg.2016.1681}

40. Solomon DH, Rassen JA, Glynn RJ, et al. The comparative safety of opioids for nonmalignant pain in older adults. Arch Intern Med. 2010;170(22):1979-1986.

41. Dhalla IA, Mamdani MM, Sivilotti ML, Kopp A, Qureshi O, Juurlink DN. Prescribing of opioid analgesics and related mortality before and after the introduction of long-acting oxycodone. CMAJ. 2009;181(12):891-896.

42. Madsen AM, Stark LM, Has P, Emerson JB, Schulkin J, Matteson KA. Opioid Knowledge and Prescribing Practices Among Obstetrician-Gynecologists. Obstet Gynecol. 2018 Jan;131(1):150-157.

43. Coleman JJ. Rescheduling Hydrocodone Combination Products: Addressing the Abuse of America's Favorite Opioid. ASAM.

https://www.asam.org/resources/publications/magazine/read/article/2015/04/10/resched uling-hydrocodone-combination-products-addressing-the-abuse-of-america-s-favoriteopioid. Accessed July 12, 2018. 
44. Bohnert AS, Valenstein M, Bair MJ, et al. Association between opioid prescribing patterns and opioid overdose-related deaths. Jama. Apr 6 2011;305(13):1315-1321.

45. Jones JD, Mogali S, Comer SD. Polydrug abuse: A review of opioid and benzodiazepine combination use. Drug and alcohol dependence. 9/1/ 2012;125(1-2):818.

46. Paulozzi LJ, Zhang K, Jones CM, Mack KA. Risk of adverse health outcomes with increasing duration and regularity of opioid therapy. Journal of the American Board of Family Medicine : JABFM. May-Jun 2014;27(3):329-338.

47. Gwira Baumblatt JA, Wiedeman C, Dunn JR, Schaffner W, Paulozzi LJ, Jones TF. High-risk use by patients prescribed opioids for pain and its role in overdose deaths. JAMA internal medicine. May 2014;174(5):796-801.

48. Washington State Agency Medical Directors' Group. Interagency Guideline on Prescribing Opioids for Pain 2015. Available from: http://www.agencymeddirectors.wa.gov/Files/2015AMDGOpioidGuideline.pdf.

49. Alexander GC, Frattaroli S, Gielen AC, eds. The Prescription Opioid Epidemic: An Evidence-Based Approach. Johns Hopkins Bloomberg School of Public Health, Baltimore, Maryland: 2015. Available from: https://www.drugabuse.gov/nidamedmedical-health-professionals/opioid-pain-management-cmes/unaccredited-module-1safe-prescribing-pain. Accessed: July 24, 2018.

50. Dowell D, Haegerich TM, Chou R. CDC Guideline for Prescribing Opioids for Chronic Pain - United States, 2016. MMWR Recommendations and Reports: Morbidity and Mortality Weekly Report. 2016;65(1):1-49. PMID: 26987082.

51. Bartels K, Mayes LM, Dingmann C, Bullard KJ, Hopfer CJ, Binswanger IA. Opioid use and storage patterns by patients after hospital discharge following surgery. PloS one. 2016;11(1):e0147972. PMID: 26824844; PMCID: PMC4732746. 
52. Bates C, Laciak R, Southwick A, Bishoff J. Overprescription of postoperative narcotics: a look at postoperative pain medication delivery, consumption and disposal in urological practice. J Urol. 2011;185(2):551-5. PMID: 21168869.

53. Chapman T, Kim N, Maltenfort M, Ilyas AM. Prospective evaluation of opioid consumption following carpal tunnel release surgery. Hand. 2017;12(1):39-42. PMID: 28082841; PMCID: PMC5207284.

54. Kim N, Matzon JL, Abboudi J, Jones C, Kirkpatrick W, Leinberry CF, et al. A prospective evaluation of opioid utilization after upper-extremity surgical procedures: identifying consumption patterns and determining prescribing guidelines. JBJS American. 2016;98(20):e89. PMID: 27869630.

55. Madsen AM, Stark LM, Has P, Emerson JB, Schulkin J, Matteson KA. Opioid Knowledge and Prescribing Practices Among Obstetrician-Gynecologists. Obstet Gynecol. 2018 Jan;131(1):150-157. PubMed PMID:29215508.

56. Paulozzi LJ, Strickler GK, Kreiner PW, Koris CM, Centers for Disease C, Prevention. Controlled Substance Prescribing Patterns--Prescription Behavior Surveillance System, Eight States, 2013. Morbidity and mortality weekly report. Surveillance summaries. Oct 16 2015;64(9):1-14.

57. Neuman MD, Bateman BT, Wunsch $\mathrm{H}$, Postoperative pain management and opioids 2 Inappropriate opioid prescription after surgery. The Lancet. Vol 393, Issue 10180, 13-19 April 2019, Pg 1547-1557. 
Figure 1. Flow Chart of the Study Population.

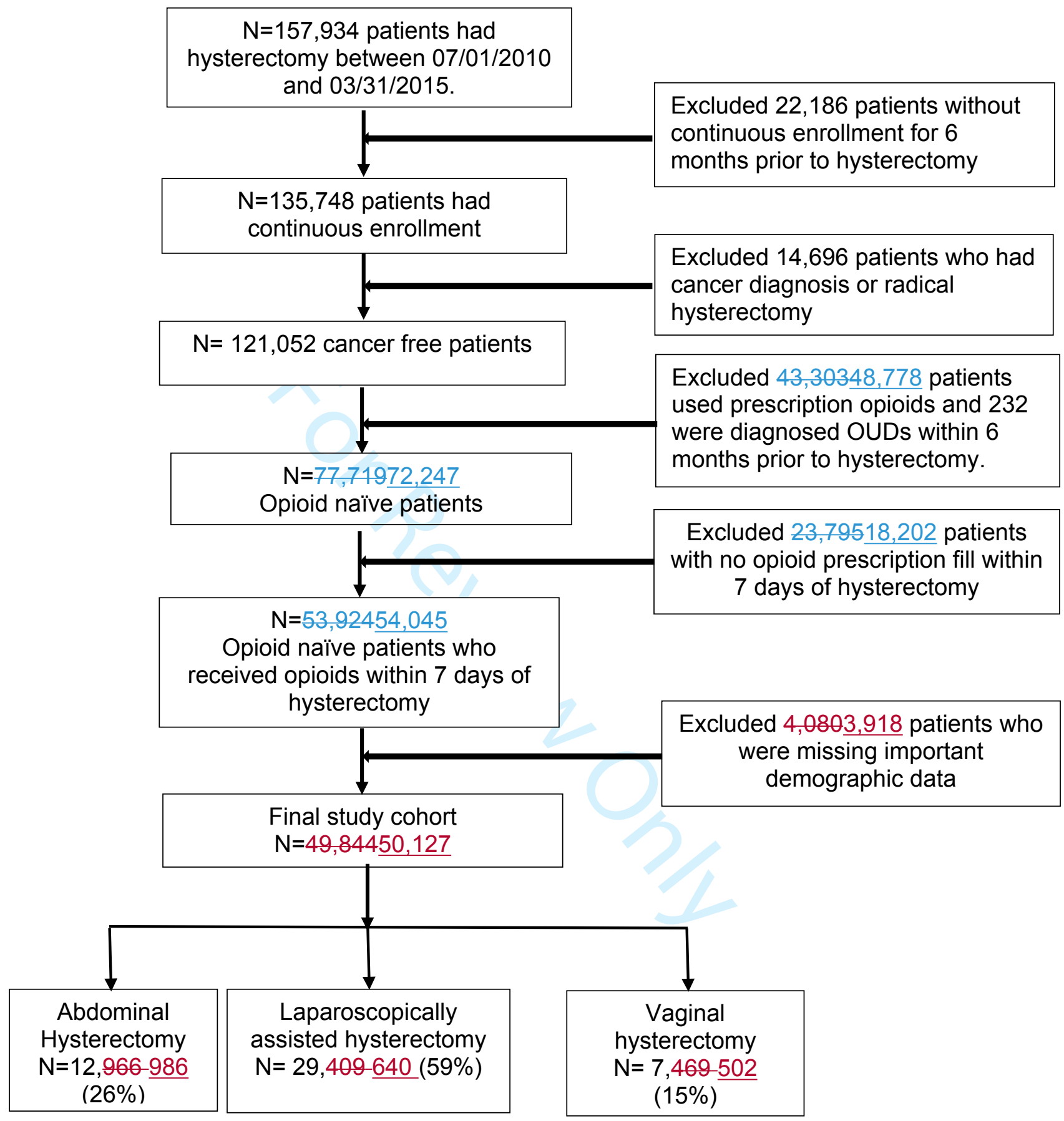


Figure 2. Trajectories of filling opioid prescriptions in 6 months post hysterectomy. The solid or dashed lines represent the distinct estimated opioid prescription filling trajectories. The dot symbols represent the mean estimated proportion in each trajectory group dispensed an opioid prescription at each month. These indicate the mean estimated probability of filling an opioid prescription for patients in each group at each time point. Overall counts and percentages of patients in each group are presented in the underneath table. Patients in trajectory 4 were classified as opioid chronic users. Patients in trajectories 1,2,3 were classified as opioid non-chronic users. The x-axis indicates each 30-day interval during the 6-month follow-up period, while the $y$-axis indicates the estimated proportion of patients filled a prescription opioid within each 30-day interval.

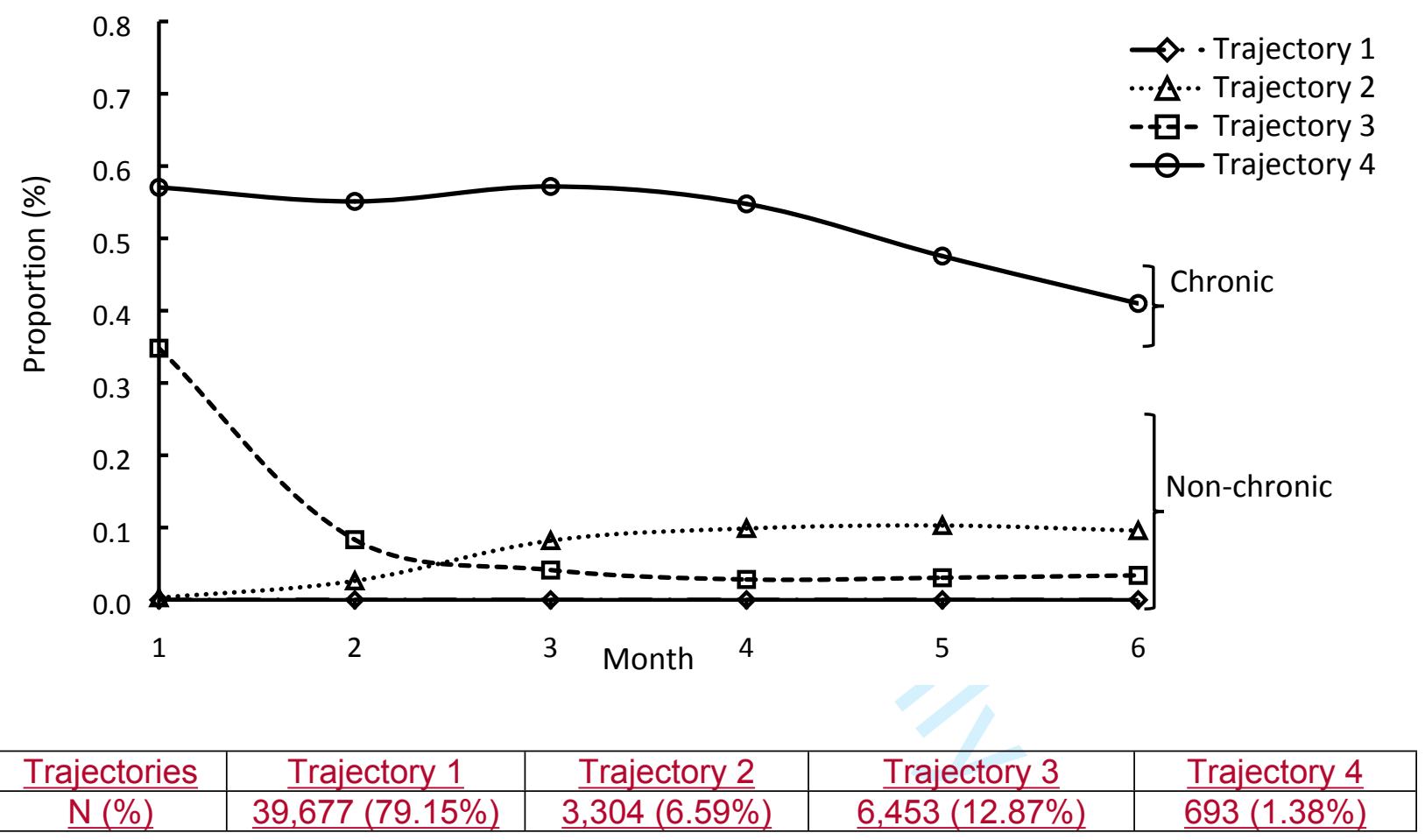


Figure 2. Proportion in each trajectory group dispensed an opioid prescription in each month.

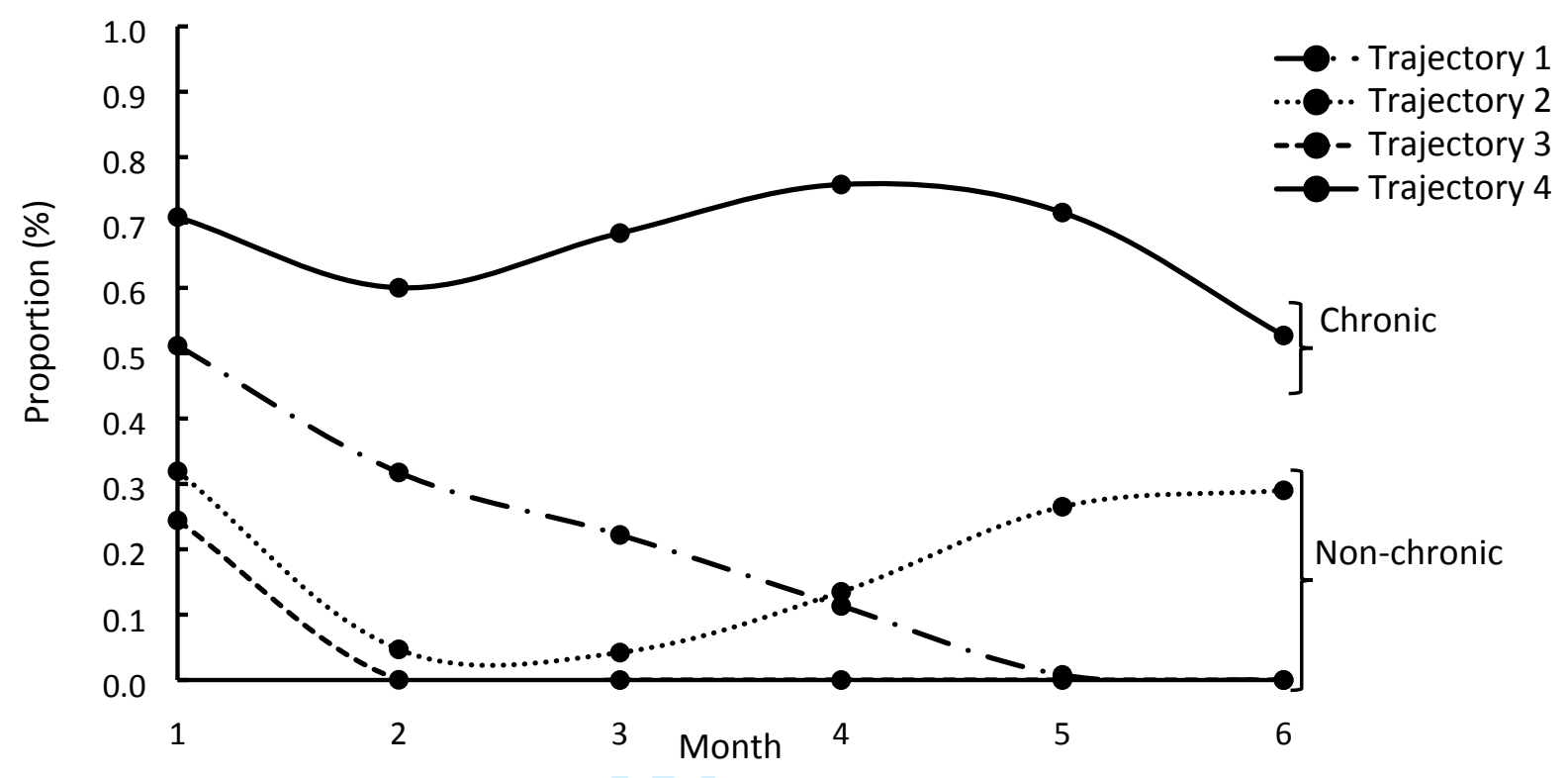

\begin{tabular}{|c|c|c|c|c|}
\hline Trajectories & 4 & 2 & 3 & 4 \\
\hline $\mathrm{N}(\%)$ & $2,808(5.63 \%)$ & $2,543(5.10 \%)$ & $44,059(88.39 \%)$ & $434(0.87 \%)$ \\
\hline
\end{tabular}

Table 1. Comparison of baseline patient characteristics among opioid-naïve patients who received prescription opioids within 7 days after hysterectomy, $\mathrm{N}=50,127$ 


\begin{tabular}{|c|c|c|c|}
\hline$\underline{\text { Patient characteristics }}$ & $\frac{\frac{\text { Non-chronic }}{\text { Users }^{+}}}{(\mathrm{N}=48,434)}$ & $\frac{\text { Chronic Opioid Users }^{\ddagger}}{(\mathrm{N}=693)}$ & $\underline{\text { P-value }}$ \\
\hline Charlson Comorbidity Index, mean (95\% Cl) & $1.60(1.57,1.64)$ & $1.74(1.45,2.02)$ & 0.3479 \\
\hline Length of stay in days, mean $(95 \% \mathrm{Cl})$ & $2.32(2.30,2.35)$ & $3.79(3.39,4.19)$ & $<0.0001$ \\
\hline Age, years, mean $(95 \% \mathrm{Cl})$ & $48.1(48.0,48.2)$ & $49.8(48.9,50.7)$ & 0.0004 \\
\hline $\begin{array}{l}\frac{\text { Age at hysterectomy, years }}{\frac{<40}{40-49}} \\
\frac{50+}{4}\end{array}$ & $\frac{\frac{9,100(18.41)}{22,538(45.59)}}{17,796(36.00)}$ & $\frac{\frac{126(18.18)}{255(36.80)}}{\underline{312(45.02)}}$ & $\leq .0001$ \\
\hline $\begin{array}{l}\frac{\text { Substance use and abuse, N (\%) }}{\text { Tobacco }} \\
\frac{\text { Alcohol }}{\text { Other substance use disorders }}\end{array}$ & $\frac{2,637(5.33)}{\frac{177(0.36)}{55(0.11)}}$ & $\frac{60(8.66)}{\frac{9(1.30)}{4(0.58)}}$ & $\begin{array}{l}\frac{0.0001}{<.0001} \\
0.0004\end{array}$ \\
\hline $\begin{array}{l}\frac{\text { Psychiatric medications, } \mathrm{N}(\%)}{\text { Benzodiazepines }} \\
\frac{\text { Antidepressants }}{\text { Stimulants }} \\
\end{array}$ & $\frac{\frac{4,201(8.50)}{9,984(20.20)}}{\underline{767(1.55)}}$ & $\frac{\frac{125(18.04)}{233(33.62)}}{\underline{25(3.61)}}$ & $\begin{array}{l}<.0001 \\
\leq .0001 \\
\leq .0001\end{array}$ \\
\hline $\begin{array}{l}\frac{\text { Pain conditions, } \mathrm{N}(\%)}{\text { Fibromyalgia }} \\
\text { Headache syndromes } \\
\text { Back pain }\end{array}$ & $\frac{1,530(3.10)}{\frac{4,019(8.13)}{4,659(9.42)}}$ & $\frac{\frac{30(4.33)}{82(11.83)}}{110(15.87)}$ & $\begin{array}{l}\frac{0.0632}{0.0004} \\
\leq .0001\end{array}$ \\
\hline $\begin{array}{c}\frac{\text { Type of hysterectomy, } \mathrm{N}(\%)}{\text { Abdominal hysterectomy }} \\
\text { Vaginal hysterectomy } \\
\text { Laparoscopic/Robotic }\end{array}$ & $\frac{12,757(25.81)}{\frac{7,428(15.03}{29,249(59.17)}}$ & $\frac{228(32.90)}{\frac{74(10.68)}{391(56.42)}}$ & $\leq .0001$ \\
\hline $\begin{array}{l}\frac{\text { Surgery setting, N (\%) }}{\text { Inpatient }} \\
\text { Outpatient }\end{array}$ & $\frac{19,805(40.06)}{29,629(59.94)}$ & $\frac{352(50.79)}{341(49.21)}$ & $\leq .0001$ \\
\hline $\begin{array}{l}\frac{\text { US census region, } N(\%)}{\text { Midwest }} \\
\frac{\text { Northeast }}{\text { South }} \\
\text { West } \\
\end{array}$ & $\frac{\frac{13,705(27.72)}{\frac{5,075(10.27)}{23,642(47.83)}}}{\underline{7,012(14.18)}}$ & $\frac{\frac{186(26.84)}{66(9.52)}}{\underline{\frac{632(47.91)}{109(15.73)}}}$ & $\underline{0.6351}$ \\
\hline $\begin{array}{l}\frac{\text { Insurance type, N (\%) }}{\text { Commercial }} \\
\text { Medicare }\end{array}$ & $\frac{45,107(91.25)}{4,327(8.75)}$ & $\frac{586(84.56)}{107(15.44)}$ & $\leq .0001$ \\
\hline $\begin{array}{l}\text { Type of health plan, } \mathrm{N}(\%) \\
\text { Exclusive provider organization } \\
\text { Health maintenance organization } \\
\text { Point of service } \\
\text { Others } \\
\end{array}$ & $\begin{array}{l}\frac{5,990(12.12)}{4,913(9.94)} \\
\frac{34,004(68.79)}{4,527(9.16)}\end{array}$ & $\begin{array}{l}\frac{70(10.10)}{89(12.84)} \\
\frac{449(64.79)}{85(12.27)} \\
\end{array}$ & $\underline{0.0008}$ \\
\hline $\begin{array}{c}\frac{\text { Year of hysterectomy }}{2010-2013} \\
2014-2015 \\
\end{array}$ & $\frac{21,949(44.40)}{27,485(55.60)}$ & $\frac{287(41.41)}{406(58.59)}$ & $\underline{0.1161}$ \\
\hline
\end{tabular}

http://mc.manuscriptcentral.com/pds 


\begin{tabular}{|c|c|c|c|}
\hline Days' supply, days & $\underline{4.85(4.82,4.87)}$ & $5.12(4.89,5.34)$ & 0.0205 \\
\hline Average daily MME, mg/day & $54.7(54.3,55.0)$ & $58.4(54.6,62.2)$ & 0.0571 \\
\hline $\begin{array}{c}\frac{\text { Type of Opioid, } \mathrm{N}(\%)}{\text { Hydrocodone }} \\
\frac{\text { Oxycodone }}{\text { Other }}\end{array}$ & $\frac{\frac{17,555(35.51)}{27,434(55.50)}}{\underline{4,445(8.99)}}$ & $\frac{\frac{266(38.38)}{354(51.08)}}{\underline{73(10.53)}}$ & $\underline{0.0553}$ \\
\hline $\begin{array}{l}\frac{\text { Tertile MME, mg/day }}{<=40} \\
\frac{<0.5-58.9}{60.0+} \\
\end{array}$ & $\frac{\frac{16,154(32.68)}{16,718(33.82)}}{\underline{16,562(33.50)}}$ & $\begin{array}{l}\frac{219(31.60)}{221(31.89)} \\
\underline{253(36.51)} \\
\end{array}$ & $\underline{0.2425}$ \\
\hline $\begin{array}{c}\frac{\text { Days' supply, days }}{1-3} \\
\frac{4-7}{8+} \\
\end{array}$ & $\frac{\frac{15,667(31.69)}{29,098(58.86)}}{\underline{4,669(9.44)}}$ & $\frac{\frac{193(27.85)}{423(61.04)}}{\underline{77(11.11)}}$ & $\underline{0.0566}$ \\
\hline
\end{tabular}

Abbreviation: MME=Morphine Milligram Equivalent

${ }^{\dagger}$ Non-chronic users refer to patients in trajectory 1, 2, and 3 in Figure 2.

${ }^{\ddagger}$ Chronic Opioid users refer to patients in trajectory 4 in Figure 2.

§Other substance use includes cocaine, marijuana, anxiolytic, stimulant, hallucinogenic drugs, or abuse of unspecified drugs/medications.

IOthers include Indemnity and Preferred provider organization. 
Table 2. Baseline and initial opioid prescription characteristics that predict chronic opioid use following hysterectomy among opioid naive patients, 2010-2015; N=50, 127

\begin{tabular}{|c|c|c|}
\hline Characteristics & Unadjusted OR (95\% CI) & Adjusted $\mathrm{OR}^{+}(95 \% \mathrm{Cl})$ \\
\hline $\begin{array}{c}\frac{\text { Age group, years }}{40-49} \\
<40 \\
\geq 50 \\
\end{array}$ & $\begin{array}{l}\frac{\text { Ref. }}{1.22(0.99,1.52)} \\
\underline{1.55(1.31,1.83)} \\
\end{array}$ & $\frac{{ }_{\text {Ref. }}}{\underline{1.20(0.97,1.49)}}$ \\
\hline $\begin{array}{c}\frac{\text { Insurance type }}{\text { Commercial }} \\
\text { Medicare } \\
\end{array}$ & $1.90 \frac{\text { Ref. }_{(1.55,}}{2.35)}$ & $1.98 \frac{\text { Ref. }}{(1.37,2.87}$ \\
\hline $\begin{array}{l}\frac{\text { Type of health plan, } \mathrm{N}(\%)}{\text { Exclusive provider organization }} \\
\text { Health maintenance organization } \\
\text { Point of service } \\
\text { Others }{ }^{\ddagger} \\
\end{array}$ & $\frac{\frac{\text { Ref. }}{1.55(1.13,2.13)}}{\frac{1.13(0.88,1.46)}{1.61(1.17,2.21)}}$ & $\frac{\frac{\text { Ref. }_{.}}{1.19(0.84,1.68)}}{\underline{1.10(0.85,1.42)}}$ \\
\hline $\begin{array}{l}\text { Type of hysterectomy } \\
\text { Vaginal hysterectomy } \\
\text { Laparoscopic/Robotic } \\
\text { Abdominal hysterectomy } \\
\end{array}$ & $\begin{array}{l}\frac{\text { Ref. }}{1.79(1.38,2.34)} \\
\underline{1.34(1.05,1.72)} \\
\end{array}$ & $\begin{array}{l}\frac{\text { Ref. }}{1.52(1.16,2.00)} \\
\underline{1.40(1.09,1.80)} \\
\end{array}$ \\
\hline Year (2010-2013 vs 2014-2015) & $1.13(0.97,1.32)$ & $1.24(1.06,1.46)$ \\
\hline Length of stay (days) & $1.14(1.11,1.16)$ & $1.12(1.10,1.15)$ \\
\hline Charlson comorbidity index & $1.18(1.06,1.31)$ & $1.16(1.04,1.30)$ \\
\hline $\begin{array}{c}\text { Pain conditions } \\
\text { Headache (Yes vs. No) } \\
\text { Back pain (Yes vs. No) }\end{array}$ & $\frac{1.52(1.20,1.91)}{1.81(1.48,2.23)}$ & $\frac{1.33(1.04,1.69)}{1.57(1.27,1.94)}$ \\
\hline $\begin{array}{c}\frac{\text { Substance use }}{\text { Alcohol }} \\
\text { Tobacco } \\
\end{array}$ & $\begin{array}{l}\underline{3.66(1.87,7.18)} \\
\underline{1.68(1.29,2.20)} \\
\end{array}$ & $\begin{array}{l}1.84(0.91,3.74) \\
1.47(1.12,1.93) \\
\end{array}$ \\
\hline$\frac{\frac{\text { Psychiatric medications }}{\text { Antidepressants }}}{\frac{\text { Benzodiazepines }}{\text { Psychostimulants }}}$ & $\frac{2.00(1.71,2.35)}{\frac{2.37(1.95,2.88)}{2.38(1.58,3.56)}}$ & $\begin{array}{l}\frac{1.71(1.45,2.03)}{1.89(1.54,2.33)} \\
\underline{1.84(1.21,2.80)} \\
\end{array}$ \\
\hline Initial & Prescription Characteristic & \\
\hline $\begin{array}{c}\frac{\text { Type of opioid }}{\text { Oxycodone }} \\
\frac{\text { Hydrocodone }}{\text { Other }} \\
\end{array}$ & $\frac{\underline{\text { Ref. }}}{\underline{1.17(1.00,1.38)}}$ & $\frac{\underline{\text { Ref. }}}{1.31(1.10,1.57)}$ \\
\hline $\begin{array}{c}\text { Days' supply, days } \\
\leq 3 \\
4-7 \\
\geq 8 \\
\end{array}$ & $\begin{array}{l}\frac{\text { Ref. }}{1.18(0.99,1.40)} \\
\underline{1.34(1.03,1.75)} \\
\end{array}$ & $\frac{\underline{\text { Ref. }}}{1.28(1.06,1.54)}$ \\
\hline $\begin{array}{c}\frac{\text { Tertile MME, mg/day }}{<40} \\
\frac{20-58.9}{\geq 60.0} \\
\end{array}$ & $\frac{\underline{\text { Ref. }}}{\underline{0.98(0.81,1.18)}}$ & $\frac{\underline{\text { Ref. }}}{\underline{1.14(0.93,1.40)}}$ \\
\hline
\end{tabular}

Abbreviation: MME=Morphine Milligram Equivalent

Adjusted model: c-statistic $=0.70$; Hosmer-Lemeshow Goodness-of-fit Test: $X^{2}=19.30, d f=8, p=0.0133$

http://mc.manuscriptcentral.com/pds 
${ }^{\dagger}$ Adjusted for baseline patient demographic and clinical characteristics listed in this table. ${ }^{\ddagger}$ Others include Indemnity and Preferred provider organization. 
Table 3. Distribution of possible indications for chronic opioid use during the 6-month follow-up period after hysterectomy: shown as N (\%).

\begin{tabular}{|c|c|c|c|c|}
\hline Possible indications & $\begin{array}{c}\text { All patients in } \\
\begin{array}{c}\text { cohort } \\
(\mathrm{N}=50,127)\end{array}\end{array}$ & $\begin{array}{c}\frac{\text { Non-chronic }}{\text { Users }} \\
\underline{(N=49,434)}\end{array}$ & $\begin{array}{c}\frac{\text { Chronic Opioid }}{\text { Users }} \\
\underline{(N=693)}\end{array}$ & P-value \\
\hline Chronic pancreatitis & $70(0.14)$ & $63(0.13)$ & $7(1.01)$ & $<.0001$ \\
\hline Headache syndromes & $3,284(6.55)$ & $\underline{3,196(6.47)}$ & $88(12.70)$ & $\leq .0001$ \\
\hline Back pain & $\underline{4,477(8.93)}$ & $\underline{4,287(8.67)}$ & $190(27.42)$ & $\leq .0001$ \\
\hline Fibromyalgia & $1,470(2.93)$ & $1,407(2.85)$ & $63(9.09)$ & $<.0001$ \\
\hline$\frac{\text { Additional anesthetic }}{\text { procedures }^{\dagger}}$ & & & & $\leq .0001$ \\
\hline$\underline{0}$ & $\underline{46,132(92.03)}$ & $\underline{45,768(92.58)}$ & $364(52.53)$ & \\
\hline$\underline{1}$ & $\underline{3,310(6.60)}$ & $\underline{3,099(6.27)}$ & $\underline{211(30.45)}$ & \\
\hline$\underline{2}$ & $525(1.05)$ & $459(0.93)$ & $66(9.52)$ & \\
\hline$\geq 3$ & $160(0.32)$ & $108(0.22)$ & $52(7.50)$ & \\
\hline
\end{tabular}

${ }^{\dagger}$ Number of additional procedures requiring anesthesia. 
Table 1. Comparison of baseline patient characteristics among opioid-naïve patients who received prescription opioids within 7 days after hysterectomy, $\mathrm{N}=49,844$

\begin{tabular}{|c|c|c|c|}
\hline Patient characteristics & $\begin{array}{c}\text { Non-chronic Users** } \\
(N=49,410)\end{array}$ & $\begin{array}{c}\text { Chronic Opioid } \\
\text { Users* } \\
(\mathrm{N}=434)\end{array}$ & P-value \\
\hline Chartson Comorbidity Index, mean (95\% Cl) & $0.4(0.4,0.4)$ & $0.8(0.7,0.9)$ & $\leftarrow .0001$ \\
\hline tength of stay in days, mean $(95 \% \mathrm{CH})$ & $1.00(1.00,1.02)$ & $2.19(1.86,2.51)$ & $<.0001$ \\
\hline Age, years, mean $(95 \% \mathrm{CH})$ & $48.1(48.0,48.2)$ & $50.5(49.3,51.7)$ & $<.0001$ \\
\hline $\begin{array}{l}\text { Age at hysterectomy, years } \\
<40 \\
40-49 \\
50+\end{array}$ & $\begin{array}{l}9,245(18.71) \\
22,461(45.46) \\
17,704(35.83)\end{array}$ & $\begin{array}{l}84(19.35) \\
141(32.49) \\
209(48.16)\end{array}$ & $<.0001$ \\
\hline $\begin{array}{l}\text { Substance use and abuse, } \mathrm{N}(\%) \\
\text { Tobacce } \\
\text { Alcohol } \\
\text { Marijuana } \\
\text { Cocaine } \\
\text { Other substance abuse*** }\end{array}$ & $\begin{array}{l}2,714(5.49) \\
181(0.37) \\
5(0.01) \\
8(0.02) \\
35(0.07)\end{array}$ & $\begin{array}{l}49(11.29) \\
8(1.84) \\
0(0.00) \\
1(0.23) \\
0(0.0)\end{array}$ & $\begin{array}{l}\leftarrow .0001 \\
.0003 \\
\text { NA } \\
0.08 \\
\text { NA }\end{array}$ \\
\hline $\begin{array}{l}\text { Psychiatric medications, } \mathrm{N}(\%) \\
\text { Benzodiazepines } \\
\text { Antidepressants } \\
\text { Stimulants } \\
\end{array}$ & $\begin{array}{c}4,403(8.91) \\
10,331(20.91) \\
766(1.55)\end{array}$ & $\begin{array}{c}87(20.05) \\
159(36.64) \\
18(4.15) \\
\end{array}$ & $\begin{array}{l}<.0001 \\
<.0001 \\
<.0001 \\
\end{array}$ \\
\hline $\begin{array}{l}\text { Pain conditions, } \mathrm{N}(\%) \\
\text { Fibromyalgia } \\
\text { Headache syndromes } \\
\text { Back pain } \\
\end{array}$ & $\begin{array}{c}1,692(3.42) \\
4,212(8.52 \\
5,053(10.23) \\
\end{array}$ & $\begin{array}{l}32(7.37) \\
41(9.45) \\
85(19.59)\end{array}$ & $\begin{array}{c}<.0001 \\
0.49 \\
<.0001 \\
\end{array}$ \\
\hline $\begin{array}{c}\text { Type of hysterectomy, } \mathrm{N}(\%) \\
\text { Abdominal hysterectomy } \\
\text { Vaginal hysterectomy } \\
\text { Laparoscopic/Robotic }\end{array}$ & $\begin{array}{c}12,814(25.93) \\
7,412(15.00) \\
29,184(59.06)\end{array}$ & $\begin{array}{l}152(35.02) \\
57(13.13) \\
225(51.84)\end{array}$ & $<.0001$ \\
\hline $\begin{array}{l}\text { US census region, } \mathrm{N}(\%) \\
\text { Midwest } \\
\text { Northeast } \\
\text { South } \\
\text { West }\end{array}$ & $\begin{array}{c}13,907(28.15) \\
5,041(10.20) \\
23,609(47.78) \\
6,853(13.87)\end{array}$ & $\begin{array}{c}118(27.19) \\
36(8.29) \\
202(46.54) \\
78(17.97)\end{array}$ & 0.07 \\
\hline $\begin{array}{c}\text { Insurance type, } \mathrm{N}(\%) \\
\text { Commercial } \\
\text { Medicare } \\
\end{array}$ & $\begin{array}{c}44,966(91.01) \\
4,444(8.99) \\
\end{array}$ & $\begin{array}{c}354(81.57) \\
80(18.43) \\
\end{array}$ & $<.0001$ \\
\hline $\begin{array}{l}\text { Type of health plan, } \mathrm{N}(\%) \\
\text { Exclusive provider organization } \\
\text { Health maintenance organization } \\
\text { Point of service } \\
\text { Others } * * * * \\
\end{array}$ & $\begin{array}{c}5,905(11.95) \\
4,899(9.91) \\
33,992(68.80) \\
4,614(9.31)\end{array}$ & $\begin{array}{c}43(9.91) \\
55(12.67) \\
274(63.13) \\
62(14.29)\end{array}$ & 0.0004 \\
\hline $\begin{array}{l}\text { Year of hysterectomy } \\
2010-2013 \\
2014-2015\end{array}$ & $\begin{array}{l}21,952(44.43) \\
27,458(55.57)\end{array}$ & $\begin{array}{l}180(41.47) \\
254(58.53)\end{array}$ & 0.22 \\
\hline \multicolumn{4}{|c|}{ Initial Opioid Prescription Characteristics } \\
\hline Days' supply, days & $4.81(4.78,4.83)$ & $5.44(5.07,5.80)$ & $<0.0001$ \\
\hline Average daily MME*****, $\mathrm{mg} /$ day & $55.0(54.7,55.4)$ & $61.1(55.5,66.8)$ & 0.03 \\
\hline $\begin{array}{c}\text { Type of Opioid, } \mathrm{N}(\%) \\
\text { Hydrocodone }\end{array}$ & $18,738(37.92)$ & $176(40.55)$ & 0.16 \\
\hline
\end{tabular}




\begin{tabular}{|c|c|c|c|}
\hline $\begin{array}{l}\text { Oxycodone } \\
\text { Other }\end{array}$ & $\begin{array}{c}27,332(55.32) \\
3,340(6.76)\end{array}$ & $\begin{array}{c}222(51.15) \\
36(8.29) \\
\end{array}$ & \\
\hline $\begin{array}{l}\text { Tertile MAME*****, mg/day } \\
\quad<=40 \\
40.5-58.9 \\
60.0+\end{array}$ & $\begin{array}{l}16,964(34.33) \\
15,794(31.97) \\
16,652(33.70)\end{array}$ & $\begin{array}{l}140(32.26) \\
124(28.57) \\
170(39.17)\end{array}$ & 0.05 \\
\hline $\begin{array}{c}\text { Days' supply, days } \\
1-3 \\
4-7 \\
8+\end{array}$ & $\begin{array}{c}15,838(32.05) \\
29,097(58.89) \\
4,475(9.06)\end{array}$ & $\begin{array}{c}116(26.73) \\
259(59.68) \\
59(13.59)\end{array}$ & 0.001 \\
\hline
\end{tabular}

Note:

* Chronic Opioid users refer to patients in trajectory 4 in Figure 2.

** Non-chronic users refer to patients in trajectory 1, 2, and 3 in Figure 2.

*** Other substance use includes anxiolytic, stimulant, hallucinogenic drugs, or abuse of unspecified drugs/medications.

**** Others including Indemnity and Preferred provider organization. ***** MME=Morphine Milligram Equivalent 
Fable 2. Baseline and initial opioid prescription characteristics that predict chronic opioid use following hysterectomy among opioid naive patients, 2010-2015; $\mathrm{N}=49,844$

\begin{tabular}{|c|c|c|}
\hline Characteristics & Unadjusted OR (95\% CI) & Adjusted OR* $(95 \% \mathrm{Cl})$ \\
\hline $\begin{array}{c}\text { Age group, years } \\
40-49 \\
<40 \\
50+\end{array}$ & $\begin{array}{c}\text { Ref. } \\
1.45(1.10,1.90) \\
1.88(1.52,2.33)\end{array}$ & $\begin{array}{c}\text { Ref. } \\
1.42(1.08,1.87) \\
1.54(1.22,1.95)\end{array}$ \\
\hline $\begin{array}{l}\text { US census region } \\
\text { Northeast } \\
\text { Midwest } \\
\text { South } \\
\text { West }\end{array}$ & $\begin{array}{c}\text { Ref. } \\
1.19(0.82,1.73) \\
1.20(0.84,1.71) \\
1.59(1.07,2.37)\end{array}$ & $\begin{array}{c}\text { Ref. } \\
1.33(0.91,1.96) \\
1.31(0.91,1.88) \\
1.77(1.18,2.66)\end{array}$ \\
\hline $\begin{array}{l}\text { Insurance type } \\
\text { Commercial } \\
\text { Medicare }\end{array}$ & $\begin{array}{c}\text { Ref. } \\
2.29(1.79,2.92)\end{array}$ & $\begin{array}{c}\text { Ref. } \\
1.58(1.19,2.11)\end{array}$ \\
\hline $\begin{array}{l}\text { Type of hysterectomy } \\
\text { Vaginal hysterectomy } \\
\text { Laparoscopic/Robotic } \\
\text { Abdominal hysterectomy }\end{array}$ & $\begin{array}{c}\text { Ref. } \\
1.00(0.75,1.34) \\
1.54(1.14,2.09)\end{array}$ & $\begin{array}{c}\text { Ref. } \\
1.03(0.77,1.39) \\
1.35(0.98,1.87)\end{array}$ \\
\hline Year (2010-2013 vs 2014-2015) & $1.13(0.93,1.37)$ & $1.27(1.05,1.56)$ \\
\hline Length of stay (days) & $1.13(1.11,1.16)$ & $1.10(1.07,1.13)$ \\
\hline Charlson comorbidity index & $1.34(1.26,1.42)$ & $1.18(1.10,1.26)$ \\
\hline $\begin{array}{l}\text { Pain conditions } \\
\text { Fibromyalgia (Yes vs. No) } \\
\text { Back pain (Yes vs. No) }\end{array}$ & $\begin{array}{l}2.25(1.56,3.23) \\
2.14(1.69,2.72)\end{array}$ & $\begin{array}{l}1.58(1.08,2.31) \\
1.70(1.32,2.18)\end{array}$ \\
\hline $\begin{array}{c}\text { Substance use } \\
\text { Alcohol } \\
\text { Tobacco } \\
\end{array}$ & $\begin{array}{l}5.11(2.50,10.44) \\
2.19(1.62,2.96) \\
\end{array}$ & $\begin{array}{l}2.28(1.06,4.87) \\
1.81(1.33,2.46)\end{array}$ \\
\hline $\begin{array}{c}\text { Psychiatric medications } \\
\text { Antidepressants } \\
\text { Benzodiazepines } \\
\text { Psychostimulants }\end{array}$ & $\begin{array}{l}2.19(1.80,2.66) \\
z .56(2.02,3.25) \\
z .75(1.71,4.43)\end{array}$ & $\begin{array}{l}1.78(1.44,2.19) \\
1.94(1.51,2.49) \\
2.07(1.26,3.41)\end{array}$ \\
\hline \multicolumn{3}{|c|}{ Initial Opioid Prescription Characteristics } \\
\hline $\begin{array}{l}\text { Type of opioid } \\
\text { Oxycodone } \\
\text { Hydrocodone } \\
\text { Other }\end{array}$ & $\begin{array}{c}\text { Ref. } \\
1.15(0.95,1.41) \\
1.33(0.93,1.89) \\
\end{array}$ & $\begin{array}{c}\text { Ref. } \\
1.35(1.07,1.70) \\
1.37(0.95,1.97) \\
\end{array}$ \\
\hline $\begin{array}{c}\text { Days' supply, days } \\
<=3 \\
4-7 \\
8+ \\
\end{array}$ & $\begin{array}{c}\text { Ref. } \\
1.22(0.96,1.51) \\
1.80(1.31,2.47) \\
\end{array}$ & $\begin{array}{c}\text { Ref. } \\
1.41(1.11,1.79) \\
2.04(1.43,2.90) \\
\end{array}$ \\
\hline $\begin{array}{l}\text { Tertile MME, mg/day } \\
<=40 \\
40.5-58.9 \\
60.0+ \\
\end{array}$ & $\begin{array}{c}\text { Ref. } \\
0.95(0.75,1.21) \\
1.24(0.99,1.55)\end{array}$ & $\begin{array}{c}\text { Ref. } \\
1.24(0.95,1.62) \\
1.70(1.28,2.26)\end{array}$ \\
\hline
\end{tabular}

Adjusted model: $c$-statistic $=0.73$; Hosmer-Lemeshow Goodness-of-fit Test: $X^{2}=9.32, d f=8, p=0.32$

*: Adjusted for baseline patient demographic and clinical characteristics listed in this table. 
Fable 3. Distribution of possible indications for chronic opioid use during the 6 -month follow-up period after hysterectomy: shown as $\mathrm{N}(\%)$.

\begin{tabular}{|c|c|c|c|c|}
\hline Possible indications & $\begin{array}{c}\text { All patients in } \\
\text { cohort } \\
(\mathrm{N}=49,844)\end{array}$ & $\begin{array}{c}\text { Non-chronic } \\
\text { Users } \\
(\mathrm{N}=49,410)\end{array}$ & $\begin{array}{c}\text { Chronic } \\
\text { Opioid Users } \\
(\mathbb{N}=414)\end{array}$ & P-value \\
\hline Rheumatoid arthritis & $6(0.01)$ & $6(0.01)$ & $\theta(0.00)$ & NA \\
\hline Chronic pancreatitis & $73(0.15)$ & $67(0.14)$ & $6(1.38)$ & $<.0001$ \\
\hline Sickle cell disease & $7(0.01)$ & $7(0.01)$ & $\theta(0.00)$ & NA \\
\hline Headache syndromes & $3,373(6.77)$ & $3,311(6.70)$ & $62(14.29)$ & $<0.0001$ \\
\hline Backpain & $4,652(9.33)$ & $4,511(9.13)$ & $141(32.49)$ & $<0.0001$ \\
\hline Fibromyalgia & $1,588(3.19)$ & $1,548(3.13)$ & $40(9.22)$ & $<0.0001$ \\
\hline $\begin{array}{l}\text { Additional anesthetic } \\
\text { procedures* }\end{array}$ & & & & $<0.0001$ \\
\hline$\theta$ & $45,840(91.97)$ & $45,621(92.33)$ & $219(50.46)$ & \\
\hline 1 & $3,316(6.65)$ & $3,177(6.43)$ & $139(32.03)$ & \\
\hline$z$ & $524(1.05)$ & $479(0.97)$ & $-45(10.37)$ & \\
\hline $3+$ & $164(0.33)$ & $133(0.27)$ & $31(7.14)$ & \\
\hline
\end{tabular}

Note:

*: Number of additional procedures requiring anesthesia. 


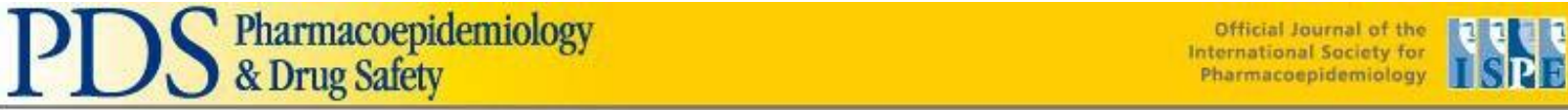

\section{CONFLICT OF INTEREST DISCLOSURE}

The Editors of Pharmacoepidemiology and Drug Safety recognize that most studies in pharmacoepidemiology cost money and thus pose a potential conflict of interest. As a conflict of interest may affect the assessment or judgment of an author, we ask that all authors (not just the Corresponding Author) complete the following form.

For Co-authors: Please complete questions 4-10. Completed forms should be saved, and emailed as an attachment to the Corresponding Author.

For Corresponding Authors: Please complete all questions. It is the responsibility of the Corresponding Author to submit completed forms on behalf of all co-authors via Manuscript Central at the point of manuscript submission.

\section{Corresponding author only (Co-authors go to Question 4): POTENTIAL STUDY INTERPRETATION CONFLICTS}

1. Some or all of the data that were used in this study were provided by a company with a vested interest in the product being studied. No

2. The sponsor of this project had the right of commenting but the authors retained the right to accept or reject comments or suggestions.

No

3. The sponsor of this project had the right of final editing and/or approval of the manuscript submitted.

No

\section{Corresponding author and Co-authors:}

POTENTIAL FINANCIAL CONFLICTS

4. I, my spouse, or one of my dependent children is an employee of a company whose product is being studied.

No

5. I, my spouse, or one of my dependent children has significant equity interest (>USD 10,000) in the company that owns the product being studied. No 
1

2

3

4

5

6

7

8

9

6. In the past three years I have:

been paid as a consultant (or in a similar capacity) by a company with a vested interest in the product being studied, on issues related to the product being studied; No been paid as a consultant (or in a similar capacity by a company with a vested interest in the product being studies, on issues unrelated to the product being studied; No received research or educational support from a company with a vested interest in the product(s) being studied.

7. A company whose product is being studied has provided funding to support the work on this project.

No

If you have answered YES to any of the above questions, or if you have additional personal, commercial or academic conflicts of interest, please draft a statement to publish with the article. e.g., AB has been reimbursed by Safe Drug Ltd. for international conference attendance.

\section{N/A}

8. Manuscript title (first six words are sufficient)

\section{Chronic Opioid Use in Women following Hysterectomy}

9. Author's full name (a separate form must be submitted for each author)

\section{Xuerong Wen}

10. In checking this box, I confirm I have completed this form to the best of myknowledge. $\square$

This form is available online by clicking here 


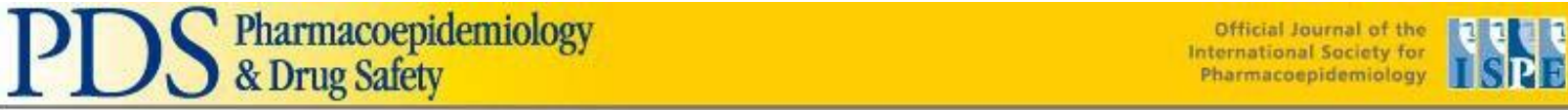

\section{CONFLICT OF INTEREST DISCLOSURE}

The Editors of Pharmacoepidemiology and Drug Safety recognize that most studies in pharmacoepidemiology cost money and thus pose a potential conflict of interest. As a conflict of interest may affect the assessment or judgment of an author, we ask that all authors (not just the Corresponding Author) complete the following form.

For Co-authors: Please complete questions 4-10. Completed forms should be saved, and emailed as an attachment to the Corresponding Author.

For Corresponding Authors: Please complete all questions. It is the responsibility of the Corresponding Author to submit completed forms on behalf of all co-authors via Manuscript Central at the point of manuscript submission.

\section{Corresponding author only (Co-authors go to Question 4): POTENTIAL STUDY INTERPRETATION CONFLICTS}

1. Some or all of the data that were used in this study were provided by a company with a vested interest in the product being studied. $\mathrm{n} / \mathrm{a}$

2. The sponsor of this project had the right of commenting but the authors retained the right to accept or reject comments or suggestions.

$\mathrm{n} / \mathrm{a}$

3. The sponsor of this project had the right of final editing and/or approval of the manuscript submitted.

$n / a$

\section{Corresponding author and Co-authors:}

POTENTIAL FINANCIAL CONFLICTS

4. I, my spouse, or one of my dependent children is an employee of a company whose product is being studied.

No

5. I, my spouse, or one of my dependent children has significant equity interest (>USD 10,000) in the company that owns the product being studied. No 
1

2

3

4

5

6

7

8

9

6. In the past three years I have:

been paid as a consultant (or in a similar capacity) by a company with a vested interest in the product being studied, on issues related to the product being studied; No

been paid as a consultant (or in a similar capacity by a company with a vested interest in the product being studies, on issues unrelated to the product being studied; No received research or educational support from a company with a vested interest in the product(s) being studied.

No

7. A company whose product is being studied has provided funding to support the work on this project.

No

If you have answered YES to any of the above questions, or if you have additional personal, commercial or academic conflicts of interest, please draft a statement to publish with the article. e.g., AB has been reimbursed by Safe Drug Ltd. for international conference attendance.

8. Manuscript title (first six words are sufficient)

Chronic Opioid Use in Women following Hysterectomy

9. Author's full name (a separate form must be submitted for each author)

\section{Kristen A. Matteson}

10. In checking this box, I confirm I have completed this form to the best of my knowledge. $\square$

This form is available online by clicking here 


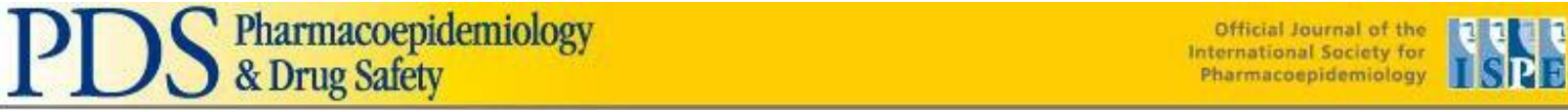

\section{CONFLICT OF INTEREST DISCLOSURE}

The Editors of Pharmacoepidemiology and Drug Safety recognize that most studies in pharmacoepidemiology cost money and thus pose a potential conflict of interest. As a conflict of interest may affect the assessment or judgment of an author, we ask that all authors (not just the Corresponding Author) complete the following form.

For Co-authors: Please complete questions 4-10. Completed forms should be saved, and emailed as an attachment to the Corresponding Author.

For Corresponding Authors: Please complete all questions. It is the responsibility of the Corresponding Author to submit completed forms on behalf of all co-authors via Manuscript Central at the point of manuscript submission.

\section{Corresponding author only (Co-authors go to Question 4): POTENTIAL STUDY INTERPRETATION CONFLICTS}

1. Some or all of the data that were used in this study were provided by a company with a vested interest in the product being studied. $n / a$

2. The sponsor of this project had the right of commenting but the authors retained the right to accept or reject comments or suggestions.

$\mathrm{n} / \mathrm{a}$

3. The sponsor of this project had the right of final editing and/or approval of the manuscript submitted.

$n / a$

\section{Corresponding author and Co-authors:}

POTENTIAL FINANCIAL CONFLICTS

4. I, my spouse, or one of my dependent children is an employee of a company whose product is being studied.

No

5. I, my spouse, or one of my dependent children has significant equity interest (>USD 10,000) in the company that owns the product being studied. No 
6. In the past three years I have:

- been paid as a consultant (or in a similar capacity) by a company with a vested interest in the product being studied, on issues related to the product being studied; No

- been paid as a consultant (or in a similar capacity by a company with a vested interest in the product being studies, on issues unrelated to the product being studied; No

- received research or educational support from a company with a vested interest in the product(s) being studied.

No

7. Acompany whose product is being studied has provided funding to support the work on this project.

No

If you have answered YES to any of the above questions, or if you have additional personal, commercial or academic conflicts of interest, please draft a statement to publish with the article. e.g., AB has been reimbursed by Safe Drug Ltd. for international conference attendance.

8. Manuscript title (first six words are sufficient)

Chronic Opioid Use in Women following Hysterectomy

9. Author's full name (a separate form must be submitted for each author) Hilary Aroke

10. In checking this box, I confirm I have completed this form to the best of my knowledge. $\square$

This form is available online by clicking here 


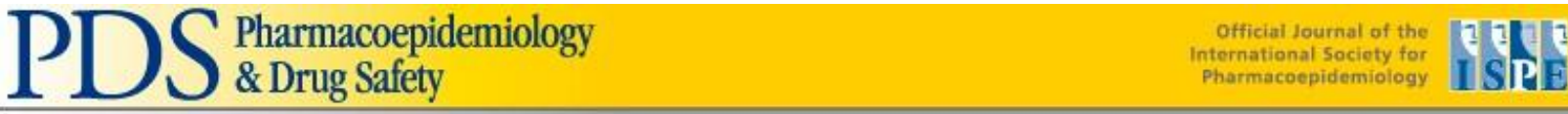

\section{CONFLICT OF INTEREST DISCLOSURE}

The Editors of Pharmacoepidemiology and Drug Safety recognize that most studies in pharmacoepidemiology cost money and thus pose a potential conflict of interest. As a conflict of interest may affect the assessment or judgment of an author, we ask that all authors (not just the Corresponding Author) complete the following form.

For Co-authors: Please complete questions 4-10. Completed forms should be saved, and emailed as an attachment to the Corresponding Author.

For Corresponding Authors: Please complete all questions. It is the responsibility of the Corresponding Author to submit completed forms on behalf of all co-authors via Manuscript Central at the point of manuscript submission.

\section{Corresponding author only (Co-authors go to Question 4): POTENTIAL STUDY INTERPRETATION CONFLICTS}

1. Some or all of the data that were used in this study were provided by a company with a vested interest in the product being studied. $\mathrm{n} / \mathrm{a}$

2. The sponsor of this project had the right of commenting but the authors retained the right to accept or reject comments or suggestions.

$\mathrm{n} / \mathrm{a}$

3. The sponsor of this project had the right of final editing and/or approval of the manuscript submitted.

$n / a$

\section{Corresponding author and Co-authors:}

POTENTIAL FINANCIAL CONFLICTS

4. I, my spouse, or one of my dependent children is an employee of a company whose product is being studied.

No

5. I, my spouse, or one of my dependent children has significant equity interest (>USD 10,000) in the company that owns the product being studied. No 
6. In the past three years I have:

- been paid as a consultant (or in a similar capacity) by a company with a vested interest in the product being studied, on issues related to the product being studied; No

- been paid as a consultant (or in a similar capacity by a company with a vested interest in the product being studies, on issues unrelated to the product being studied; No

- received research or educational support from a company with a vested interest in the product(s) being studied.

No

7. Acompany whose product is being studied has provided funding to support the work on this project.

No

If you have answered YES to any of the above questions, or if you have additional personal, commercial or academic conflicts of interest, please draft a statement to publish with the article. e.g., AB has been reimbursed by Safe Drug Ltd. for international conference attendance.

8. Manuscript title (first six words are sufficient)

Chronic Opioid Use of after Hysterectomy

9. Author's full name (a separate form must be submitted for each author)

\section{Stephen J Kogut}

10. In checking this box, I confirm I have completed this form to the best of my knowledge. $\square$

This form is available online by clicking here 


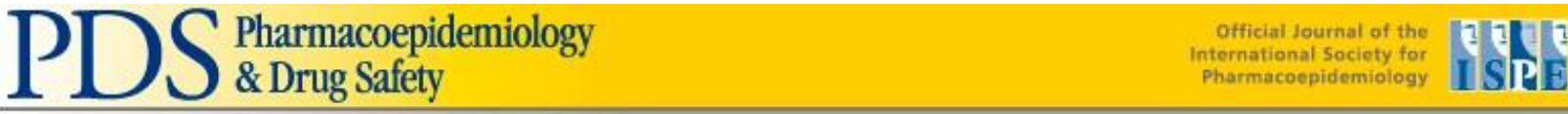

\section{CONFLICT OF INTEREST DISCLOSURE}

The Editors of Pharmacoepidemiology and Drug Safety recognize that most studies in pharmacoepidemiology cost money and thus pose a potential conflict of interest. As a conflict of interest may affect the assessment or judgment of an author, we ask that all authors (not just the Corresponding Author) complete the following form.

For Co-authors: Please complete questions 4-10. Completed forms should be saved, and emailed as an attachment to the Corresponding Author.

For Corresponding Authors: Please complete all questions. It is the responsibility of the Corresponding Author to submit completed forms on behalf of all co-authors via Manuscript Central at the point of manuscript submission.

\section{Corresponding author only (Co-authors go to Question 4): POTENTIAL STUDY INTERPRETATION CONFLICTS}

1. Some or all of the data that were used in this study were provided by a company with a vested interest in the product being studied. $\mathrm{n} / \mathrm{a}$

2. The sponsor of this project had the right of commenting but the authors retained the right to accept or reject comments or suggestions.

$\mathrm{n} / \mathrm{a}$

3. The sponsor of this project had the right of final editing and/or approval of the manuscript submitted.

$n / a$

\section{Corresponding author and Co-authors:}

POTENTIAL FINANCIAL CONFLICTS

4. I, my spouse, or one of my dependent children is an employee of a company whose product is being studied.

No

5. I, my spouse, or one of my dependent children has significant equity interest (>USD 10,000) in the company that owns the product being studied. No 
6. In the past three years I have:

- been paid as a consultant (or in a similar capacity) by a company with a vested interest in the product being studied, on issues related to the product being studied; No

- been paid as a consultant (or in a similar capacity by a company with a vested interest in the product being studies, on issues unrelated to the product being studied; No

- received research or educational support from a company with a vested interest in the product(s) being studied.

No

7. Acompany whose product is being studied has provided funding to support the work on this project.

No

If you have answered YES to any of the above questions, or if you have additional personal, commercial or academic conflicts of interest, please draft a statement to publish with the article. e.g., AB has been reimbursed by Safe Drug Ltd. for international conference attendance.

8. Manuscript title (first six words are sufficient)

Chronic Opioid Use in Women following Hysterectomy

9. Author's full name (a separate form must be submitted for each author) Lynn Taylor

10. In checking this box, I confirm I have completed this form to the best of my knowledge. $\square$ 